- Supporting Information -

Org. Lett.

\title{
Electrophilic Allylations and Benzylations of Indoles in Neutral Aqueous or Alcoholic Solutions
}

\author{
Martin Westermaier and Herbert Mayr* \\ Department Chemie und Biochemie \\ Ludwig Maximilians Universität München \\ Butenandtstr. 5-13 (Haus F), \\ 81377 München, Germany \\ Fax: +49 (0) 89218077717 \\ Email: herbert.mayr@cup.uni-muenchen.de
}


Chemicals. Acetone was distilled (bp. $56{ }^{\circ} \mathrm{C}$ ), acetonitrile (Fisher Scientific) was used as purchased, 2,2,2-trifluoroethanol (Acros) was distilled over drierite (bp. $72{ }^{\circ} \mathrm{C}$ ). Water was purified with Millipore MilliQplus. All starting materials were obtained from Aldrich, Acros, Lancaster and Merck and used without further purification. Allylic substrates were prepared from the corresponding alcohols by following published procedures: 4-chloropent-2-ene (2), ${ }^{\mathrm{S} 1}$ geranyl chloride (5a) ${ }^{\mathrm{S} 2}$ geranyl bromide $\quad(\mathbf{5 b}),{ }^{\mathrm{S} 3} \quad$ 3-chloro-1,3-diphenylpropene $\quad(\mathbf{7 a}),{ }^{\mathrm{S} 4} \quad$ 3-bromo-1,3-diphenylpropene $\quad(\mathbf{7 b}),{ }^{\mathrm{S} 3}$ 1-chloroindane (9) ${ }^{\mathrm{S} 5}$ and 5-chloromethyl-benzo[1,3]-dioxole (10). ${ }^{\mathrm{S} 6}$ 1-Methylindole (1b) was prepared according to literature. ${ }^{\mathrm{S} 7}$

Analytics. ${ }^{1} \mathrm{H}-\mathrm{NMR}$ spectra were recorded on Varian Mercury 200 (200 MHz) or Bruker ARX 300 $(300 \mathrm{MHz})$ spectrometers. Chemical shifts are reported from TMS with the solvent resonance as the internal standard $\left(\mathrm{CDCl}_{3}: \delta=7.26 \mathrm{ppm}\right)$. Data are reported as follows: chemical shift (multiplicity, coupling constants, integration intensity) with $\mathrm{s}=$ singlet, $\mathrm{d}=$ doublet, $\mathrm{t}=$ triplet, $\mathrm{q}=$ quartet, $\mathrm{br}=$ broad and $\mathrm{m}=$ multiplet. ${ }^{13} \mathrm{C}$-NMR spectra were recorded on a Bruker ARX $300(75.5 \mathrm{MHz})$ spectrometer with complete proton decoupling. Chemical shifts are reported in ppm from TMS with the solvent as internal standard $\left(\mathrm{CDCl}_{3}: \delta=77.0 \mathrm{ppm}\right)$. Spin multiplicities are derived from DEPT-135 spectra. The assignment of the peak signals to the compounds is based on 2D-NMR experiments or made by comparison with simulated spectra by the program ACD. GC-MS analysis were performed on Agilent 5973 MSD [HP-5MS-capillar column with length $30 \mathrm{~m}$, diameter $0.25 \mathrm{~mm}$, flow rate 1.0 $\mathrm{mL} / \mathrm{min}$, injector, split $(23.9 \mathrm{~mL} / \mathrm{min})$, carrier gas helium, quadrupol mass spectrometer]. Data are reported as follows: retention time and heating program [method A: $70{ }^{\circ} \mathrm{C}(2 \mathrm{~min})-25^{\circ} \mathrm{C} / \mathrm{min} \rightarrow$ $150{ }^{\circ} \mathrm{C}-50^{\circ} \mathrm{C} / \min \rightarrow 250{ }^{\circ} \mathrm{C}(12 \min )$; method B: $70{ }^{\circ} \mathrm{C}(2 \min )-25^{\circ} \mathrm{C} / \min \rightarrow 150{ }^{\circ} \mathrm{C}-50{ }^{\circ} \mathrm{C} /$ $\min \rightarrow 250{ }^{\circ} \mathrm{C}(18 \mathrm{~min})$; method $\left.\mathrm{C}: 110{ }^{\circ} \mathrm{C}(2 \mathrm{~min})-50{ }^{\circ} \mathrm{C} / \min \rightarrow 270{ }^{\circ} \mathrm{C}(5 \mathrm{~min})\right]$. Elemental analyses were carried out in the "Mikroanalytisches Labor" of the Department of Chemistry and Biochemistry of the LMU München. Chromatographic purification was done with Merck silica gel 60 (mesh $40-63 \mu \mathrm{m}$ ). $\mathrm{R}_{\mathrm{f}}$ values are given for the major isomer.

Typical Reaction Procedure. To a suspension of $\mathrm{NH}_{4} \mathrm{HCO}_{3}(10 \mathrm{mmol})$ and indole derivative (1a or 1b, $25 \mathrm{mmol})$ in aqueous acetone or aqueous acetonitrile $(25 \mathrm{~mL})$, the allyl or benzyl halide (5.0 mmol) was added. After the solution was stirred at ambient temperature for the time specified in Tables 2 and 3, water was added $(30 \mathrm{~mL})$ and the organic phase was separated. The aqueous phase was extracted with $\mathrm{Et}_{2} \mathrm{O}(3 \times 30 \mathrm{~mL})$. The combined organic phases were dried $\left(\mathrm{MgSO}_{4}\right)$ and the solvents removed in the vacuum. Indole (1a, bp. $\left.103-107{ }^{\circ} \mathrm{C}, 3 \times 10^{-3} \mathrm{mbar}\right)$ or 1-methylindole (1) bp. $95-98{ }^{\circ} \mathrm{C}, 3 \times 10^{-3} \mathrm{mbar}$ ) was removed from the crude product by Kugelrohr distillation. The residue was purified by flash column chromatography. The 2-substituted isomer was eluted earlier than the 3-substituted isomer. 
3-[(E)-1-Methylbut-2-enyl]-1H-indole (3a). ${ }^{\mathrm{S} 8}$ 4-Chloropent-2-ene (2, $\left.209 \mathrm{mg}, 2.00 \mathrm{mmol}\right)$, indole (1a, $1.17 \mathrm{~g}, 10.0 \mathrm{mmol})$, and $\mathrm{NH}_{4} \mathrm{HCO}_{3}(316 \mathrm{mg}, 4.00 \mathrm{mmol})$ were stirred in acetone/water $(80 / 20=$ $\mathrm{v} / \mathrm{v}, 10 \mathrm{~mL}$ ) for $1 \mathrm{~h}$ to give $365 \mathrm{mg}(99 \%)$ of a mixture of $\mathbf{3 a}$ and $\mathbf{3 b}$ as a brown oil, which was separated by column chromatography $\left(\mathrm{R}_{\mathrm{f}} 0.36, n\right.$-hexane:EtOAc $\left.7: 1\right)$ to give $297 \mathrm{mg}(80 \%)$ of 3 a.

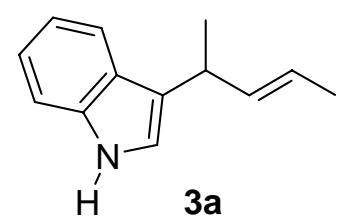

${ }^{1} \mathrm{H}-\mathrm{NMR}\left(\mathrm{CDCl}_{3}, 300 \mathrm{MHz}\right): \delta 1.42\left(\mathrm{~d}, J=7.0 \mathrm{~Hz}, 3 \mathrm{H}, 1^{\prime}-\mathrm{CH}_{3}\right), 1.66\left(\mathrm{dd}, J=6.0 \mathrm{~Hz},{ }^{4} J=1.2 \mathrm{~Hz}, 3\right.$ $\mathrm{H}, \mathrm{CH}_{3}$ ), 3.69 (dqd, ${ }^{3} J=7.0 \mathrm{~Hz}, 6.6 \mathrm{~Hz},{ }^{4} J=0.9 \mathrm{~Hz}, 1 \mathrm{H}, 1$ '-H), 5.48-5.72 (m, $\left.2 \mathrm{H},-\mathrm{HC}=\mathrm{CH}-\right), 6.88$ $(\mathrm{d}, J=2.4 \mathrm{~Hz}, 1 \mathrm{H}, 2-\mathrm{H}), 7.06-7.19(\mathrm{~m}, 3 \mathrm{H}), 7.28$ (d, $J=8.0 \mathrm{~Hz}, 1 \mathrm{H}, 7-\mathrm{H}), 7.64$ (dd, $J=8.0 \mathrm{~Hz}, J=$ $1.2 \mathrm{~Hz}, 1 \mathrm{H}, 4-\mathrm{H}), 7.78$ (br s, $1 \mathrm{H}, \mathrm{NH}) .{ }^{13} \mathrm{C}-\mathrm{NMR}\left(\mathrm{CDCl}_{3}, 75.5 \mathrm{MHz}\right): \delta 17.8$ (q, C-4'), 20.9 (q, 1'$\mathrm{CH}_{3}$ ), 33.9 (d, C-1'), 111.1 (d, C-7), 119.0 (d, C-4), 119.7 (d, C-5), 120.1 (d, C-6), 121.2 (s, C-3), 121.8 (d, C-2), 123.2 (d, C-3'), 126.8 (s, C-7a), 136.2 (d, C-2'), 136.6 (s, C-3a), NOESY, gHSQC, and gHMBC experiments were performed to assign the resonances. GC-MS (method A): $t_{R} 8.20 \mathrm{~min} ; \mathrm{m} / \mathrm{z}$ (\%) $185(51)\left[\mathrm{M}^{+}\right], 170$ (100), 168 (13), 155 (23), 154 (20), 144 (18), 128 (11), 115 (12).

3-(3-Methylbut-2-enyl)-1H-indole (11a). ${ }^{\mathrm{S} 9}$ Prenyl bromide (4b, $\left.231 \mu \mathrm{L}, 2.00 \mathrm{mmol}\right)$, indole (1a, 1.17 g, $10.0 \mathrm{mmol})$, and $\mathrm{NH}_{4} \mathrm{HCO}_{3}(316 \mathrm{mg}, 4.00 \mathrm{mmol})$ were stirred in acetone/water $(80 / 20=\mathrm{v} / \mathrm{v}$, $10 \mathrm{~mL}$ ) for $2 \mathrm{~h}$ to give $370 \mathrm{mg}$ (99\%) of a mixture of 11a and other substituted indoles as a yellow oil, which was separated by column chromatography $\left(\mathrm{R}_{\mathrm{f}} 0.28, n\right.$-hexane:EtOAc 7:1) to give $338 \mathrm{mg}$ (91\%) of 11a as a pale yellow oil.<smiles>CC(C)=CCc1c[nH]c2ccccc12</smiles>

${ }^{1} \mathrm{H}-\mathrm{NMR}\left(\mathrm{CDCl}_{3}, 300 \mathrm{MHz}\right): \delta 1.75,1.76\left(2 \times \mathrm{d},{ }^{4} J=1.2 \mathrm{~Hz}, 2 \times 3 \mathrm{H}, 2 \times 12-\mathrm{CH}_{3}\right), 3.45\left(\mathrm{~d},{ }^{3} J=7.0\right.$ $\mathrm{Hz}, 2 \mathrm{H}, 10-\mathrm{H}), 5.43$ (t sept, $\left.{ }^{3} J=7.0 \mathrm{~Hz},{ }^{4} J=1.2 \mathrm{~Hz}, 1 \mathrm{H}, 11-\mathrm{H}\right), 6.90\left(\mathrm{~d},{ }^{3} J=2.1 \mathrm{~Hz}, 1 \mathrm{H}, 2-\mathrm{H}\right)$, 7.07-7.21 (m, 2 H, 5-H, 6-H), 7.30 (d, $\left.{ }^{3} J=7.8 \mathrm{~Hz}, 1 \mathrm{H}, 7-\mathrm{H}\right), 7.59$ (d, $\left.{ }^{3} J=7.8 \mathrm{~Hz}, 1 \mathrm{H}, 4-\mathrm{H}\right), 7.79$ (br. s, $1 \mathrm{H}, 1-\mathrm{H}) .{ }^{13} \mathrm{C}-\mathrm{NMR}\left(\mathrm{CDCl}_{3}, 75.5 \mathrm{MHz}\right): \delta 17.8$ (q), 24.1 (t), 25.7 (q), 111.0 (d), $116.2(\mathrm{~s}), 119.0$ (d), 119.1 (d), 121.1 (d), 121.9 (d), 123.1 (d), 127.5 (s), 131.9 (s), 136.5 (s). GC-MS (method A): $t_{R}$

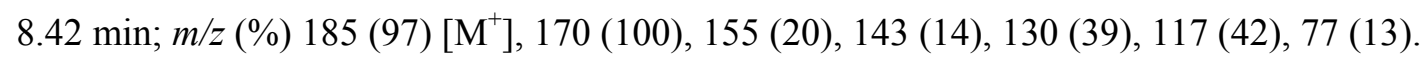

3-[(2E)-3,7-Dimethylocta-2,6-dienyl]-1H-indole (12a). ${ }^{\text {S9 }}$ Geranyl bromide (5b, $726 \mu \mathrm{L}, 5.00$ mmol), indole (1a, $2.93 \mathrm{~g}, 25.0 \mathrm{mmol})$, and $\mathrm{NH}_{4} \mathrm{HCO}_{3}(791 \mathrm{mg}, 10.0 \mathrm{mmol})$ were stirred in acetone/water $(80 / 20=\mathrm{v} / \mathrm{v}, 25 \mathrm{~mL})$ for $1 \mathrm{~h}$ to give $760 \mathrm{mg}(60 \%)$ of a mixture of 12a and 12b as a yellow oil, which was separated by column chromatography $\left(\mathrm{R}_{\mathrm{f}} 0.53, n\right.$-hexane:EtOAc $\left.5: 1\right)$ to give $713 \mathrm{mg}(56 \%)$ of 12a as a yellow oil. 


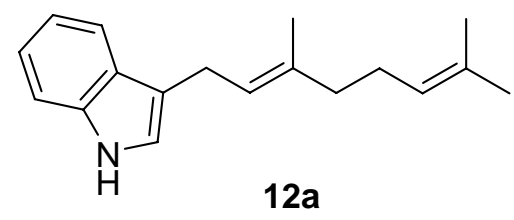

${ }^{1} \mathrm{H}-\mathrm{NMR}\left(\mathrm{CDCl}_{3}, 300 \mathrm{MHz}\right): \delta 1.60,1.68,1.75\left(3 \mathrm{s,}, 3 \times 3 \mathrm{H}, 3 \times \mathrm{CH}_{3}\right), 2.04-2.14\left(\mathrm{~m}, 4 \mathrm{H}, 2 \times \mathrm{CH}_{2}\right)$, $3.46\left(\mathrm{~d}, J=7.1 \mathrm{~Hz}, 2 \mathrm{H}, \mathrm{CH}_{2}\right), 5.10-5.14(\mathrm{~m}, 1 \mathrm{H},=\mathrm{CH}-), 5.43-5.48(\mathrm{~m}, 1 \mathrm{H},=\mathrm{CH}-), 6.92(\mathrm{br} \mathrm{s}, 1 \mathrm{H})$, 7.07-7.23 (m, $2 \mathrm{H}), 7.32$ (d, $J=8.0 \mathrm{~Hz}, 1 \mathrm{H}), 7.59$ (d, $J=8.0 \mathrm{~Hz}, 1 \mathrm{H}), 7.84$ (br. s, $1 \mathrm{H}, \mathrm{NH}) .{ }^{13} \mathrm{C}-$ NMR ( $\left.\mathrm{CDCl}_{3}, 75.5 \mathrm{MHz}\right): \delta 16.1(\mathrm{q}), 17.7$ (q), $24.0(\mathrm{t}), 25.7(\mathrm{q}), 26.7(\mathrm{t}), 39.7(\mathrm{t}), 111.0(\mathrm{~d}), 116.2(\mathrm{~s})$, 119.0 (d), 119.1 (d), 121.2 (d), 121.9 (d), 122.9 (d), 124.4 (d), 127.5 (s), 131.4 (s), 135.6 (s), 136.5 (s). GC-MS (method A): $t_{R} 11.12 \mathrm{~min} ; \mathrm{m} / z(\%) 253\left(\mathrm{M}^{+}, 68\right), 184$ (100), 182 (48), 170 (54), 168 (47), 155 (15), 154 (15), 143 (18), 131 (22), 130 (77), 117 (35).

3-(3-Phenylallyl)-1H-indole (13a) ${ }^{\mathrm{S} 10}$ und 3-(1-phenylallyl)-1H-indole (13c). Cinnamyl bromide (6b, $763 \mathrm{mg}, 5.00 \mathrm{mmol})$, indole (1a, $2.93 \mathrm{~g}, 25.0 \mathrm{mmol})$, and $\mathrm{NH}_{4} \mathrm{HCO}_{3}(791 \mathrm{mg}, 10.0 \mathrm{mmol})$ were stirred in acetone/water $(80 / 20=\mathrm{v} / \mathrm{v}, 25 \mathrm{~mL})$ for $24 \mathrm{~h}$ to give $828 \mathrm{mg}(71 \%)$ of a mixture of 13a and 13c $(8: 1)$ as a light brown oil.<smiles>CC(C)(C)CC=Cc1ccccc1</smiles><smiles>C=CC(c1ccccc1)c1c[nH]c2ccccc12</smiles>

13a: ${ }^{1} \mathrm{H}-\mathrm{NMR}\left(200 \mathrm{MHz}, \mathrm{CDCl}_{3}\right): \delta 3.66\left(\mathrm{~d},{ }^{3} \mathrm{~J}=5.5 \mathrm{~Hz}, 2 \mathrm{H}, \mathrm{CH}_{2}\right), 6.42-6.58(\mathrm{~m}, 2 \mathrm{H}), 6.93(\mathrm{~s}, 1 \mathrm{H})$, $7.02-7.39(\mathrm{~m}, 8 \mathrm{H}), 7.63\left(\mathrm{~d},{ }^{3} J=7.6 \mathrm{~Hz}, 1 \mathrm{H}\right), 7.83(\mathrm{br} \mathrm{s}, 1 \mathrm{H}, \mathrm{NH})$. GC-MS (method A): $t_{R} 12.72 ; \mathrm{m} / z$ (\%) $233(100)\left[\mathrm{M}^{+}\right], 232$ (56), 206 (17), 156 (24), 130 (42), 115 (24).

13c: ${ }^{1} \mathrm{H}-\mathrm{NMR}\left(200 \mathrm{MHz}, \mathrm{CDCl}_{3}\right): \delta 3.64\left(\mathrm{~d},{ }^{3} J=7.6 \mathrm{~Hz}, 1 \mathrm{H}\right), 4.88(\mathrm{dd}, J=12 \mathrm{~Hz}, J=7.6 \mathrm{~Hz}, 1 \mathrm{H})$, 6.28-6.60 (m, 2 H), 6.98 (s, $1 \mathrm{H}), 7.13-7.38$ (m, 8 H), 7.65 (d, J = 8.0 Hz, $1 \mathrm{H}), 7.82$ (br s, $1 \mathrm{H}, \mathrm{NH})$.

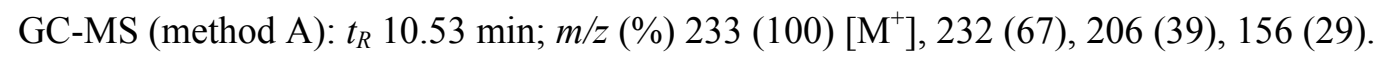

3-(1,3-Diphenylallyl)-1H-indole (14a). ${ }^{\mathrm{S} 8}$ 3-Bromo-1,3-diphenylpropene (7b, $\left.1.37 \mathrm{~g}, 5.00 \mathrm{mmol}\right)$, indole (1a, $2.93 \mathrm{~g}, 25.0 \mathrm{mmol})$, and $\mathrm{NH}_{4} \mathrm{HCO}_{3}(791 \mathrm{mg}, 10.0 \mathrm{mmol})$ were stirred in acetone/water $(80 / 20=\mathrm{v} / \mathrm{v}, 25 \mathrm{~mL})$ for $48 \mathrm{~h}$ to give $928 \mathrm{mg}(60 \%)$ of a mixture of $\mathbf{1 4 a}$ and $\mathbf{1 4} \mathbf{b}$, which was separated by column chromatography $\left(\mathrm{R}_{\mathrm{f}} 0.65, n\right.$-hexane:EtOAc $\left.7: 1\right)$ to give $866 \mathrm{mg}$ (56\%) of 14a as a colorless oil. 
<smiles>C(=Cc1c[nH]c2ccccc12)c1c[nH]c2ccccc12</smiles>

${ }^{1} \mathrm{H}-\mathrm{NMR}\left(200 \mathrm{MHz}, \mathrm{CDCl}_{3}\right): \delta 5.06\left(\mathrm{~d},{ }^{3} J=7.5 \mathrm{~Hz}, 1 \mathrm{H}, 10-\mathrm{H}\right), 6.34-6.42(\mathrm{~m}, 1 \mathrm{H}, 12-\mathrm{H}), 6.63-6.67$ (m, 1 H, 11-H), 6.80-7.01 (m, 3 H), 7.01-7.21 (m, 12 H), 7.91 (br. s, 1 H, 1-H). GC-MS (method C): $t_{R} 9.53 \mathrm{~min} ; \mathrm{m} / z(\%) 232(100)\left[\mathrm{M}^{+}-\mathrm{Ph}\right], 219$ (14), 117 (58), 90 (17).

3-Cyclohex-2-enyl-1H-indole (15a). ${ }^{\mathrm{S} 8}$ 3-Bromocyclohexene (8, $\left.805 \mathrm{mg}, 5.00 \mathrm{mmol}\right)$, indole (1a, 2.93 g, $25.0 \mathrm{mmol})$, and $\mathrm{NH}_{4} \mathrm{HCO}_{3}(791 \mathrm{mg}, 10.0 \mathrm{mmol})$ were stirred in $\mathrm{CH}_{3} \mathrm{CN} /$ water $(90 / 10=\mathrm{v} / \mathrm{v}, 25$ $\mathrm{mL}$ ) for $30 \mathrm{~min}$ to give $690 \mathrm{mg}(70 \%)$ of $\mathbf{1 5 a}$ as a yellow oil.<smiles>CC(C)(C)[Mg]</smiles>

${ }^{1} \mathrm{H}-\mathrm{NMR}\left(300 \mathrm{MHz}, \mathrm{CDCl}_{3}\right): \delta 1.56-2.12\left(\mathrm{~m}, 3 \times 2 \mathrm{H}, 3 \times \mathrm{CH}_{2}\right), 3.70-3.75(\mathrm{~m}, 1 \mathrm{H}, 1$ '-H), 5.84-5.86 (m, $\left.2 \times 1 \mathrm{H}, 11-\mathrm{H}, 3^{\prime}-\mathrm{H}\right), 6.90\left(\mathrm{~d},{ }^{4} J=2.0 \mathrm{~Hz}, 2-\mathrm{H}\right), 7.07\left(\mathrm{dt},{ }^{3} J=7.5 \mathrm{~Hz},{ }^{4} J=1.5 \mathrm{~Hz}, 1 \mathrm{H}, 6-\mathrm{H}\right), 7.18$ $\left(\mathrm{dt},{ }^{3} J=7.5 \mathrm{~Hz},{ }^{4} J=1.5 \mathrm{~Hz}, 1 \mathrm{H}, 5-\mathrm{H}\right), 7.31\left(\mathrm{dd},{ }^{3} J=8.0 \mathrm{~Hz},{ }^{4} J=0.9 \mathrm{~Hz}, 1 \mathrm{H}, 7-\mathrm{H}\right), 7.65\left(\mathrm{dd},{ }^{3} J=8.1\right.$ $\left.\mathrm{Hz},{ }^{4} J=1.0 \mathrm{~Hz}, 1 \mathrm{H}, 4-\mathrm{H}\right), 7.87$ (br. s, $\left.1 \mathrm{H}, 1-\mathrm{H}\right) .{ }^{13} \mathrm{C}-\mathrm{NMR}\left(75.5 \mathrm{MHz}, \mathrm{CDCl}_{3}\right.$ ): $\delta 20.8,25.3,30.2(3$ $\times$ t), 32.7 (d), 111.2 (d), 119.1 (d), 119.2 (d), 120.9 (s), 121.4 (d), 121.8 (d), 126.7 (s), 127.6 (d), 130.4

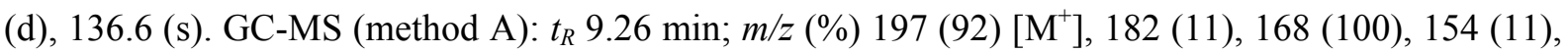
$130(12), 117(28)$.

3-(Indan-1-yl)-1H-indole (16a) and 2-(indan-1-yl)-1H-indole (16b). 1-Chloroindane (9, $763 \mathrm{mg}$, $5.00 \mathrm{mmol}$ ), indole (1a, $2.93 \mathrm{~g}, 25.0 \mathrm{mmol})$, and $\mathrm{NH}_{4} \mathrm{HCO}_{3}(791 \mathrm{mg}, 10.0 \mathrm{mmol})$ were stirred in acetone/water $(80 / 20=\mathrm{v} / \mathrm{v}, 25 \mathrm{~mL})$ for $24 \mathrm{~h}$ to give $595 \mathrm{mg}(51 \%)$ of a mixture of $\mathbf{1 6 a}$ and $\mathbf{1 6 b}(10: 1)$ as a colorless viscous oil.<smiles>CC(C)(C)c1c[nH]c2ccccc12</smiles><smiles>O=[13C]CC1c2ccccc2CC1c1cc2ccccc2[nH]1</smiles>

16a: ${ }^{1} \mathrm{H}-\mathrm{NMR}\left(300 \mathrm{MHz}, \mathrm{CDCl}_{3}\right): \delta 2.15-2.28(\mathrm{~m}, 1 \mathrm{H}), 2.52-2.63(\mathrm{~m}, 1 \mathrm{H}), 2.91-3.06(\mathrm{~m}, 2 \mathrm{H}), 4.64$ (t, $J=8.1 \mathrm{~Hz}, 1 \mathrm{H}), 6.85(\mathrm{~d}, J=2.1 \mathrm{~Hz}, 1 \mathrm{H}), 7.02-7.46(\mathrm{~m}, 8 \mathrm{H}), 7.83$ (br s, $1 \mathrm{H}, \mathrm{NH}) .{ }^{13} \mathrm{C}-\mathrm{NMR}$ (75.5 MHz, $\mathrm{CDCl}_{3}$ ): $\delta 31.7$ (t), 34.8 (t), 42.4 (d), 111.2 (d), 119.2 (d), 119.5 (d), 119.7 (s), 121.4 (d), 121.9 (d), 124.4 (d) 124.7 (d), 126.2 (d), 126.4 (d), 126.9 (s), 136.7 (s), 144.0 (s), 146.6 (s). GC-MS 
$\left(\operatorname{method~A):~} t_{R} 11.81 \mathrm{~min} ; \mathrm{m} / z(\%) 233(83)\left[\mathrm{M}^{+}\right], 232\right.$ (100), 217 (25), 116 (26). HR-EI-MS: Calcd. for $\mathrm{C}_{17} \mathrm{H}_{14} \mathrm{~N}$ : 233.3158; Found 233.1181.

3-Benzo[1,3]dioxol-5-ylmethyl-1H-indole (17a). 5-Chloromethyl-benzo[1,3]-dioxole (10, $676 \mathrm{mg}$, $5.00 \mathrm{mmol})$, indole (1a, $2.93 \mathrm{~g}, 25.0 \mathrm{mmol})$, and $\mathrm{NH}_{4} \mathrm{HCO}_{3}(791 \mathrm{mg}, 10.0 \mathrm{mmol})$ were stirred in acetone/water $(80 / 20=\mathrm{v} / \mathrm{v}, 25 \mathrm{~mL})$ for $24 \mathrm{~h}$ to give $1.08 \mathrm{~g}(86 \%)$ of a mixture of 17a and 17b $(15: 1)$, which was purified by recrystallization ( $n$-hexane:Et $\left.{ }_{2} \mathrm{O} 3: 2\right)$ to give a white solid with mp. $197{ }^{\circ} \mathrm{C}$.<smiles>c1ccc2c(Cc3ccc4c(c3)OCO4)c[nH]c2c1</smiles>

${ }^{1} \mathrm{H}-\mathrm{NMR}\left(\mathrm{CDCl}_{3}, 300 \mathrm{MHz}\right): \delta 4.03(\mathrm{~s}, 2 \mathrm{H}, 10-\mathrm{H}), 5.89$ (s, $\left.2 \mathrm{H}, 17-\mathrm{H}\right), 6.69-6.76(\mathrm{~m}, 3 \times 1 \mathrm{H}, 12-\mathrm{H}$, 13-H, 16-H), 6.93 (s, $1 \mathrm{H}, 2-\mathrm{H}), 7.07$ (t, $\left.{ }^{3} J=7.5 \mathrm{~Hz},{ }^{4} J=3,6 \mathrm{~Hz}, 1 \mathrm{H}, 6-\mathrm{H}\right), 7.18\left(\mathrm{t},{ }^{3} J=7.2 \mathrm{~Hz},{ }^{3} J=\right.$ $3.6 \mathrm{~Hz}, 1 \mathrm{H}, 5-\mathrm{H}), 7.35$ (d, $\left.{ }^{3} J=3.9 \mathrm{~Hz}, 1 \mathrm{H}, 7-\mathrm{H}\right), 7.50$ (d, $\left.{ }^{3} J=4.2 \mathrm{~Hz}, 1 \mathrm{H}, 4-\mathrm{H}\right), 7.94$ (br. s, $1 \mathrm{H}, 1-$ H). ${ }^{13} \mathrm{C}-\mathrm{NMR}\left(\mathrm{CDCl}_{3}, 75.5 \mathrm{MHz}\right): \delta 31.3(\mathrm{t}), 100.7$ (t), 108.0 (d), 109.2 (d), 111.1 (d), 116.0 (s), 119.1 (d), 119.4 (d), 121.4 (d), 122.1 (d), 122.2 (d), 127.4 (s), 135.1 (s), 136.5 (s), 145.7 (s), 147.6 (s). GC-

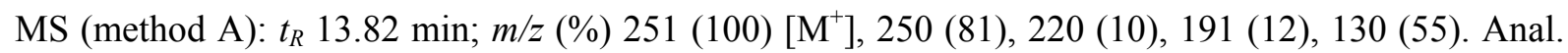
Calcd for $\left(\mathrm{C}_{16} \mathrm{H}_{13} \mathrm{NO}_{2}\right)$ : C, 76.47; H, 5.23; N, 5.58; Found: C, 76.20; H, 5.42; N, 5.52.

\section{1-Methyl-3-(1-methylbut-2-enyl)-1H-indole (18a) and 1-methyl-2-(1-methylbut-2-enyl)-1H-indol}

(18b). 4-Chloropent-2-ene (2,580 $\mu \mathrm{L}, 5.00 \mathrm{mmol}), 1$-methylindole (1b, $3.20 \mathrm{~mL}, 25.0 \mathrm{mmol})$, and $\mathrm{NH}_{4} \mathrm{HCO}_{3}(791 \mathrm{mg}, 10.0 \mathrm{mmol})$ were stirred in acetone/water $(80 / 20=\mathrm{v} / \mathrm{v}, 25 \mathrm{~mL})$ for $1 \mathrm{~h}$ to give 986 mg (99\%) of a mixture of 18a and 18b (10:1) as a yellow oil.
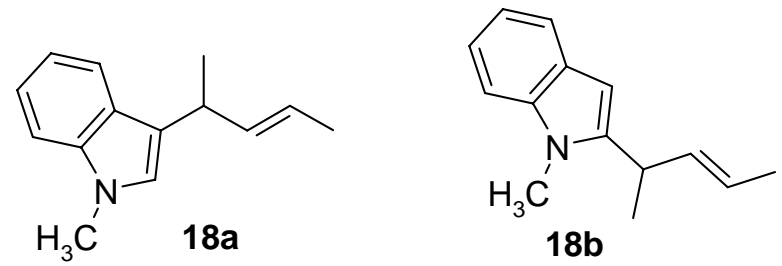

18a: ${ }^{1} \mathrm{H}-\mathrm{NMR}\left(300 \mathrm{MHz}, \mathrm{CDCl}_{3}\right): \delta 1.42\left(\mathrm{~d},{ }^{3} J=6.9 \mathrm{~Hz}, 3 \mathrm{H}\right), 1.67\left(\mathrm{dt},{ }^{3} J=6.0 \mathrm{~Hz},{ }^{4} J=1.2 \mathrm{~Hz}, 3 \mathrm{H}\right)$, 3.73 (s, $3 \mathrm{H}), 5.48-5.73(\mathrm{~m}, 2 \mathrm{H}), 6.80(\mathrm{~s}, 1 \mathrm{H}, 2-\mathrm{H}), 7.05-7.28(\mathrm{~m}, 3 \mathrm{H}), 7.62\left(\mathrm{~d},{ }^{3} J=7.8 \mathrm{~Hz}, 1 \mathrm{H}\right)$. ${ }^{13} \mathrm{C}-\mathrm{NMR}\left(75.5 \mathrm{MHz}, \mathrm{CDCl}_{3}\right.$ ): $\delta 17.8$ (q), 21.1 (q), 32.6 (d), 33.9 (q), 109.1 (d), 118.5 (d), 119.7 (d), 119.8 (s), 121.4 (d), 123.0 (d), 125.0 (d), 127.2 (s), 136.4 (d), 137.3 (s). GC-MS (method A): $t_{R} 8.09$ $\min ; m / z(\%) 199(47)\left[\mathrm{M}^{+}\right], 184(100), 168(25)$.

18b: ${ }^{1} \mathrm{H}-\mathrm{NMR}\left(300 \mathrm{MHz}, \mathrm{CDCl}_{3}\right): \delta 1.37-1.42(\mathrm{~m}, 3 \mathrm{H}), 1.64-1.68(\mathrm{~m}, 3 \mathrm{H}), 3.65(\mathrm{~s}, 3 \mathrm{H}), 5.34-5.59$ $(\mathrm{m}, 1 \mathrm{H}), 6.28(\mathrm{~s}, 1 \mathrm{H}), 6.98-7.28(\mathrm{~m}, 3 \mathrm{H}), 7.55\left(\mathrm{~d},{ }^{3} J=7.5 \mathrm{~Hz}, 1 \mathrm{H}\right)$. GC-MS (method A): $t_{R} 8.22$ $\min ; m / z(\%) 199(75)\left[\mathrm{M}^{+}\right], 184(100), 168(29)$.

Anal. Calcd for $\left(\mathrm{C}_{14} \mathrm{H}_{17} \mathrm{~N}\right)$ : C, 84.13; H, 8.58; N, 7.05; Found: C, 84.37; H, 8.60; N, 7.03. 
1-Methyl-3-(3-methylbut-2-enyl)-1H-indole $\quad(19 a)^{\mathrm{S} 9}$ und 1-methyl-2-(3-methylbut-2-enyl)-1Hindole (19b). ${ }^{\mathrm{S} 9}$ Prenyl bromide (4b, $\left.231 \mu \mathrm{L}, 2.00 \mathrm{mmol}\right)$, 1-methylindole (1b, $\left.1.28 \mathrm{~mL}, 10.0 \mathrm{mmol}\right)$, and $\mathrm{NH}_{4} \mathrm{HCO}_{3}(316 \mathrm{mg}, 4.00 \mathrm{mmol})$ were stirred in acetone/water $(80 / 20=\mathrm{v} / \mathrm{v}, 10 \mathrm{~mL})$ for $1 \mathrm{~h}$ to give $339 \mathrm{mg}(85 \%)$ of a mixture of $\mathbf{1 9 a}$ and $\mathbf{1 9 b}(10: 1)$ as a yellow liquid.
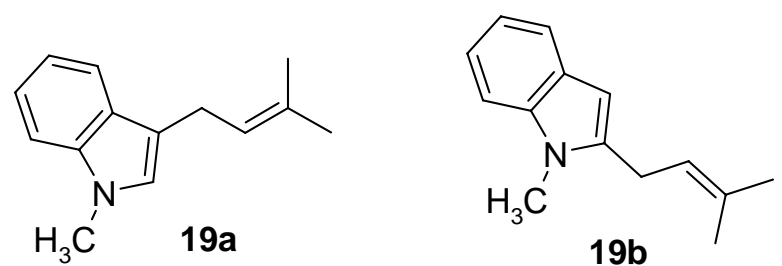

19a: ${ }^{1} \mathrm{H}-\mathrm{NMR}\left(300 \mathrm{MHz}, \mathrm{CDCl}_{3}\right): \delta 1.75\left(\mathrm{~d}, J=1 \mathrm{~Hz}, 3 \mathrm{H}, \mathrm{CH}_{3}\right), 1.76\left(\mathrm{~s}, 3 \mathrm{H}, \mathrm{CH}_{3}\right), 3.43(\mathrm{~d}, J=6.9$ $\mathrm{Hz}, 2 \mathrm{H}, \mathrm{CH}_{2}$ ), 3.70 (s, $3 \mathrm{H}, \mathrm{NCH}_{3}$ ), 5.42 (t sept, $\left.J=7.2 \mathrm{~Hz}, J=1.2 \mathrm{~Hz}, 1 \mathrm{H},=\mathrm{CH}\right), 6.78(\mathrm{~s}, 1 \mathrm{H}, 2-\mathrm{H})$, 7.05-7.27 (m, $3 \mathrm{H}), 7.58(\mathrm{~d}, J=8.1 \mathrm{~Hz}, 1 \mathrm{H}) .{ }^{13} \mathrm{C}-\mathrm{NMR}$ (75.5 MHz, $\left.\mathrm{CDCl}_{3}\right): \delta 17.8(\mathrm{q}), 24.0(\mathrm{t}), 25.7$ (q), 32.5 (q), 109.1 (d), 114.6 (s), 118.5 (d), 119.1 (d), 121.5 (d), 123.3 (d, =CH), 126.0 (d, C-2), 127.8 (s), 131.7 (s), 137.2 (s), gHSQC and gHMBC experiments were performed to assign the resonances.

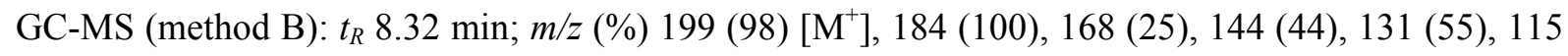
(12), 77 (9).

19b: ${ }^{1} \mathrm{H}-\mathrm{NMR}\left(300 \mathrm{MHz}, \mathrm{CDCl}_{3}\right): \delta 1.73,1.75\left(2 \times \mathrm{s}, 2 \times 3 \mathrm{H}, 2 \times 12-\mathrm{CH}_{3}\right), 3.43\left(\mathrm{~d},{ }^{3} J=6.9 \mathrm{~Hz}, 1 \mathrm{H}\right.$, 10-H), $3.63\left(\mathrm{~s}, 3 \mathrm{H}, 1-\mathrm{CH}_{3}\right), 5.30-5.39(\mathrm{~m}, 1 \mathrm{H},=\mathrm{CH}), 6.23(\mathrm{~s}, 1 \mathrm{H}, 3-\mathrm{H}), 7.02-7.27$ (m, $3 \mathrm{H}, 5-\mathrm{H}, 6-$

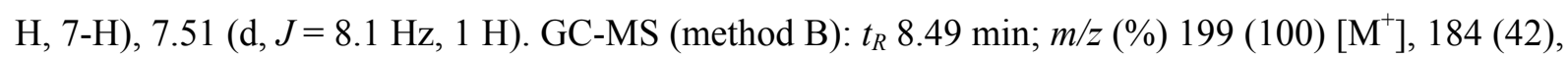
168 (17), 158 (11), 144 (50), 131 (74), 115 (12), 77 (7).

1-Methyl-3-(3,7-dimethylocta-2,6-dienyl)-1H-indole (20a). Geranyl bromide (5b, $434 \mathrm{mg}, 2.00$ mmol), 1-methylindole (1b, $1.28 \mathrm{~mL}, 10.0 \mathrm{mmol})$, and $\mathrm{NH}_{4} \mathrm{HCO}_{3}(316 \mathrm{mg}, 4.00 \mathrm{mmol})$ were stirred in acetone/water $(80 / 20=\mathrm{v} / \mathrm{v}, 10 \mathrm{~mL})$ for $1 \mathrm{~h}$ to give $365 \mathrm{mg}(71 \%)$ of a mixture of 20a and 20b as a yellow oil, which was separated by column chromatography $\left(\mathrm{R}_{\mathrm{f}} 0.65, n\right.$-hexane/EtOAc $\left.7: 1\right)$ to give $343 \mathrm{mg}(67 \%)$ of $\mathbf{2 0 a}$.

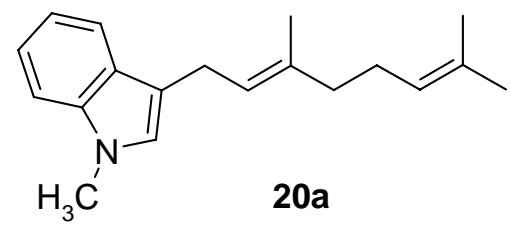

${ }^{1} \mathrm{H}-\mathrm{NMR}\left(300 \mathrm{MHz}, \mathrm{CDCl}_{3}\right): \delta 1.60,1.68,1.75\left(3 \mathrm{~s}, 3 \times 3 \mathrm{H}, 3 \times \mathrm{CH}_{3}\right), 1.97-2.17\left(\mathrm{~m}, 4 \mathrm{H}, 2 \times \mathrm{CH}_{2}\right)$, $3.45\left(\mathrm{~d}, J=7.1 \mathrm{~Hz}, 2 \mathrm{H}, \mathrm{CH}_{2}\right), 3.72\left(\mathrm{~s}, 3 \mathrm{H}, \mathrm{N}-\mathrm{CH}_{3}\right), 5.10-5.15(\mathrm{~m}, 1 \mathrm{H},=\mathrm{CH}-), 5.42-5.47(\mathrm{~m}, 1 \mathrm{H}$, $=\mathrm{CH}-), 6.78(\mathrm{~s}, 1 \mathrm{H}, 2-\mathrm{H}), 7.08-7.26(\mathrm{~m}, 3 \mathrm{H}), 7.57(\mathrm{~d}, J=8.0 \mathrm{~Hz}, 1 \mathrm{H}) .{ }^{13} \mathrm{C}-\mathrm{NMR}(75.5 \mathrm{MHz}$, $\left.\mathrm{CDCl}_{3}\right): \delta 16.0(\mathrm{q}), 17.7(\mathrm{q}), 23.9(\mathrm{t}), 25.7(\mathrm{q}), 26.6(\mathrm{t}), 32.5(\mathrm{q}), 39.7(\mathrm{t}), 109.0(\mathrm{~d}), 114.5(\mathrm{~s}), 118.5$ (d), 119.1 (d), 121.4 (d), 123.1 (d), 124.4 (d), 126.0 (d), 127.8 (s), 131.3 (s), 135.4 (s), 137.2 (s). GCMS (method A): $t_{R} 10.81 \mathrm{~min} ; \mathrm{m} / z(\%) 267(100)\left[\mathrm{M}^{+}\right], 198$ (94), 184 (57), 168 (16), 144 (71), 131 (56). HR-EI-MS: Calcd. for $\mathrm{C}_{19} \mathrm{H}_{25} \mathrm{~N}$ : 267.4178; Found 267.1997. 
1-Methyl-3-(1,3-diphenylallyl)-1H-indole (21a). ${ }^{\mathrm{S} 8}$ 3-Bromo-1,3-diphenylpropene (7b, $1.37 \mathrm{~g}, 5.00$ mmol), 1-methylindole (1b, $3.20 \mathrm{~mL}, 25.0 \mathrm{mmol})$, and $\mathrm{NH}_{4} \mathrm{HCO}_{3}(791 \mathrm{mg}, 10.0 \mathrm{mmol})$ were stirred in acetone/water $(80 / 20=\mathrm{v} / \mathrm{v}, 25 \mathrm{~mL})$ for $48 \mathrm{~h}$ to give $835 \mathrm{mg}(50 \%)$ of 21a as a yellow oil containing traces of $\mathbf{2 1 b}$.<smiles>CCCCCC(/C=C/c1ccccc1)c1cn(C)c2ccccc12</smiles>

21a: ${ }^{1} \mathrm{H}-\mathrm{NMR}\left(200 \mathrm{MHz}, \mathrm{CDCl}_{3}\right): \delta 3.73\left(\mathrm{~s}, 3 \mathrm{H}, 1-\mathrm{CH}_{3}\right), 5.11\left(\mathrm{~d},{ }^{3} \mathrm{~J}=7.6 \mathrm{~Hz}, 1 \mathrm{H}, 10-\mathrm{H}\right), 6.41-6.47$ (m, $1 \mathrm{H}), 6.69-6.77$ (m, $1 \mathrm{H}), 6.99-7.18$ (m, $1 \mathrm{H}, \mathrm{Ar}-\mathrm{H}), 7.18-7.49$ (m, $13 \mathrm{H}, \mathrm{Ar}-\mathrm{H})$. GC-MS (method C): $t_{R} 10.1 \mathrm{~min} ; \mathrm{m} / z(\%) 246(100)\left[\mathrm{M}^{+}-\mathrm{Ph}\right], 231$ (51), 117 (64).

3-Cyclohex-2-enyl-1-methyl-1H-indole (22a). ${ }^{\mathrm{S} 10}$ 3-Bromocyclohexene (8, $\left.585 \mu \mathrm{L}, 5.00 \mathrm{mmol}\right)$, 1 methylindole (1b, $3.20 \mathrm{~mL}, 25.0 \mathrm{mmol})$, and $\mathrm{NH}_{4} \mathrm{HCO}_{3}(791 \mathrm{mg}, 10.0 \mathrm{mmol})$ were stirred in $\mathrm{CH}_{3} \mathrm{CN} /$ water $(90 / 10=\mathrm{v} / \mathrm{v}, 25 \mathrm{~mL})$ for $24 \mathrm{~h}$ to give $750 \mathrm{mg}(71 \%)$ of $22 \mathrm{a}$ as a pale yellow oil.<smiles>Cn1cc(C2C=CCCC2)c2ccccc21</smiles>

${ }^{1} \mathrm{H}-\mathrm{NMR}\left(200 \mathrm{MHz}, \mathrm{CDCl}_{3}\right): \delta 1.48-1.99\left(\mathrm{~m}, 6 \mathrm{H}, 3 \times \mathrm{CH}_{2}\right), 3.55\left(\mathrm{~s}, 3 \mathrm{H}, \mathrm{NCH}_{3}\right), 3.56-3.64(\mathrm{~m}, 1 \mathrm{H})$, 5.71-5.82 (m, 2 H, $2 \times=\mathrm{CH}-), 6.67(\mathrm{~s}, 1 \mathrm{H}, 2-\mathrm{H}), 6.94-7.17$ (m, $3 \mathrm{H}), 7.53$ (d, J=7.8 Hz, $1 \mathrm{H})$. GC-

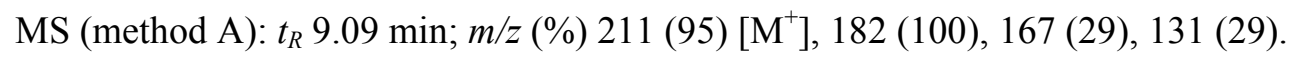

3-Benzo[1,3]dioxol-5-ylmethyl-1-methyl-1H-indole (23a). 5-Chloromethyl-benzo[1,3]-dioxole (10, $676 \mathrm{mg}, 5.00 \mathrm{mmol}$ ), 1-methylindole (1b, $3.20 \mathrm{~mL}, 25.0 \mathrm{mmol})$, and $\mathrm{NH}_{4} \mathrm{HCO}_{3}(791 \mathrm{mg}, 10.0 \mathrm{mmol})$ were stirred in acetone/water $(80 / 20=\mathrm{v} / \mathrm{v}, 25 \mathrm{~mL})$ for $24 \mathrm{~h}$ to give $744 \mathrm{mg}(56 \%)$ of a mixture of $23 \mathrm{a}$ and 23b, which was separated by column chromatography $\left(\mathrm{R}_{\mathrm{f}} 0.46, n\right.$-hexane/EtOAc $\left.7: 1\right)$ to give 702 $\operatorname{mg}(53 \%)$ of $23 a$.<smiles>Cn1cc(Cc2ccc3c(c2)OCO3)c2ccccc21</smiles>

${ }^{1} \mathrm{H}-\mathrm{NMR}\left(300 \mathrm{MHz}, \mathrm{CDCl}_{3}\right): \delta 3.72\left(\mathrm{~s}, 3 \mathrm{H}, \mathrm{NCH}_{3}\right), 4.00\left(\mathrm{~s}, 2 \mathrm{H}, \mathrm{CH}_{2}\right), 5.88\left(\mathrm{~s}, 2 \mathrm{H}, \mathrm{OCH}_{2} \mathrm{O}\right), 6.69-$ $6.76(\mathrm{~m}, 4 \mathrm{H}), 7.03-7.29(\mathrm{~m}, 3 \mathrm{H}), 7.49(\mathrm{~d}, J=7.8 \mathrm{~Hz}, 1 \mathrm{H}) .{ }^{13} \mathrm{C}-\mathrm{NMR}\left(75.5 \mathrm{MHz}, \mathrm{CDCl}_{3}\right): \delta 31.6(\mathrm{t})$, 32.9 (q, $\left.\mathrm{NCH}_{3}\right), 101.1$ (t), 108.3 (d), 109.4 (d), 109.6 (d), 114.7 (s), 119.1 (d), 119.5 (d), 121.6 (d), 121.9 (d), 127.3 (d), 128.1 (s), 135.7 (s), 137.5 (s), 146.0 (s), 147.9 (s). GC-MS (method A): $t_{R} 13.1$ 
$\min ; m / z(\%) 265(100)\left[\mathrm{M}^{+}\right], 264$ (74), 144 (83). HR-EI-MS: Calcd. for $\mathrm{C}_{19} \mathrm{H}_{25} \mathrm{~N}$ : 265.3146; Found 265.1112.

\section{References}

(S1) H. Mayr, H. Klein, G. Kolberg, Chem. Ber. 1984, 117, 2555-2579.

(S2) F. K. Sheffy, J. P. Godschalx, J. K. Stille, J. Am. Chem. Soc. 1984, 106, 4833-4840.

(S3) R. M. Coates, M. W. Johnson, J. Org. Chem. 1980, 45, 2685-2697.

(S4) T. Hayashi, A. Yamamato, Y. Ho, E. Nishioka, H. Miura, K. Yanagi, J. Am. Chem. Soc. 1989, $111,6301-6311$.

(S5) Y. El Ahmad, E. Laurent, P. Maillet, A. Talab, J. F. Teste, R. Dokhan, G. Tran, R. Ollivier, J. Med. Chem. 1997, 40, 952-960.

(S6) E. M. Schultz, R. T. Arnold, J. Am. Chem. Soc. 1949, 71, 1911-1913.

(S7) W. C. Guida, D. J. Mathre, J. Org. Chem. 1980, 45, 3172-3176.

(S8) M. Bandini, A. Melloni, A. Umani-Ronchi, Org. Lett. 2004, 6, 3199-3202.

(S9) X. Zhu, A. Ganesan, J. Org. Chem. 2002, 67, 2705-2708.

(S10) M. Ishikura, M. Kamada, I. Oda, T. Ohta, M. Terashima, J. Heterocycl. Chem. 1987, 24, 377386. 

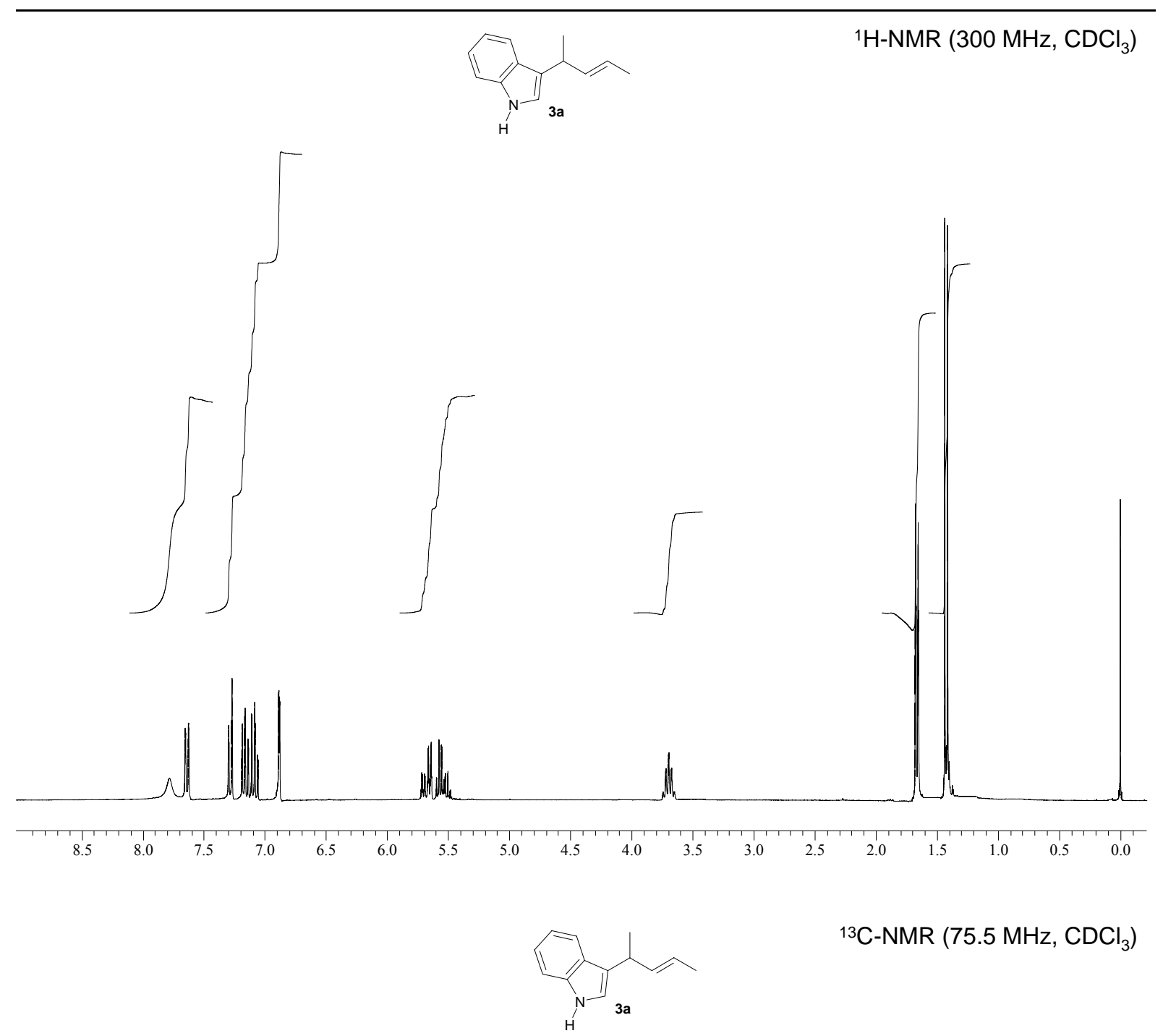

${ }^{13} \mathrm{C}-\mathrm{NMR}\left(75.5 \mathrm{MHz}, \mathrm{CDCl}_{3}\right)$

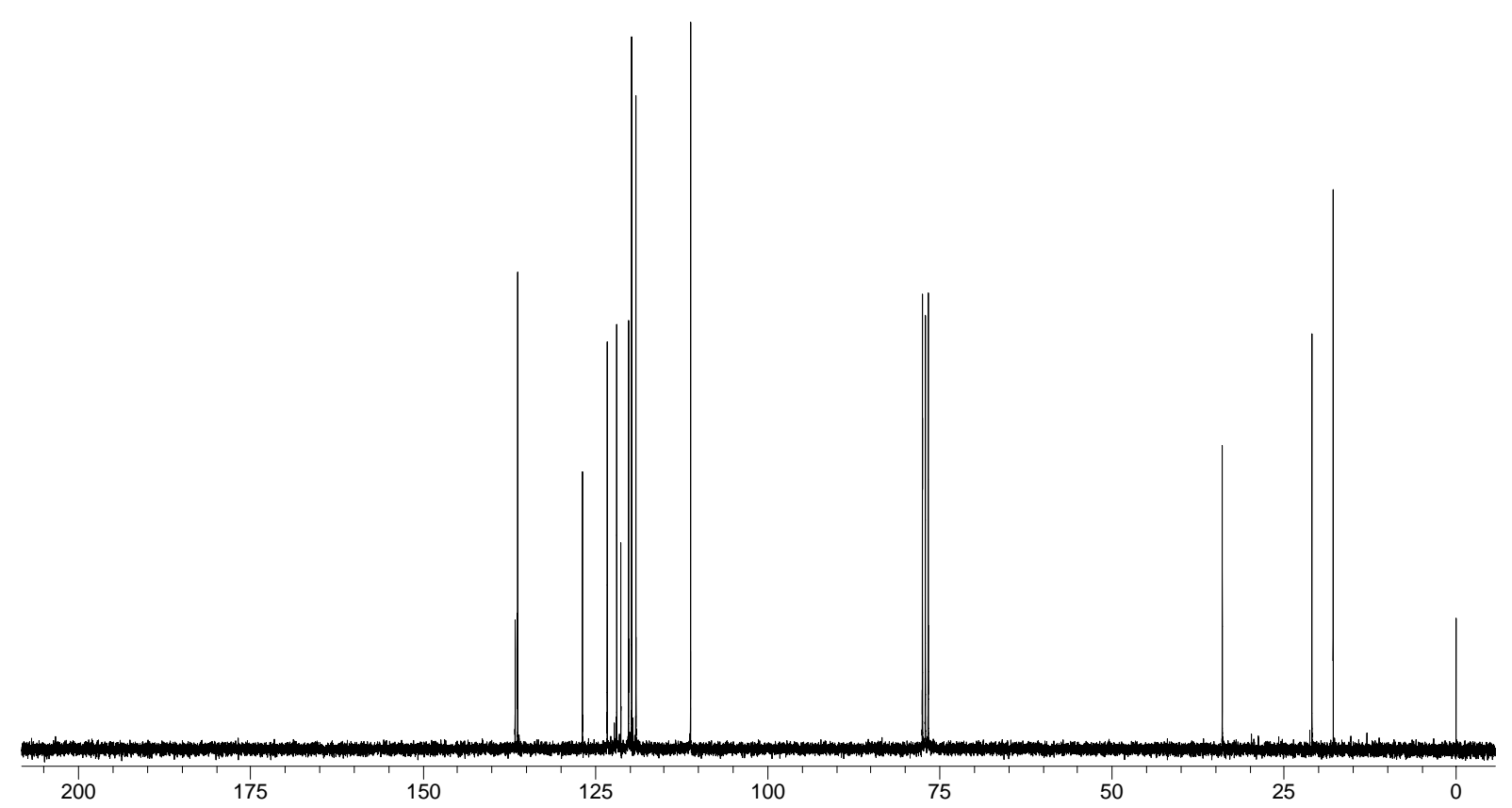




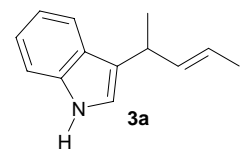

DEPT $\left(75.5 \mathrm{MHz}, \mathrm{CDCl}_{3}\right)$

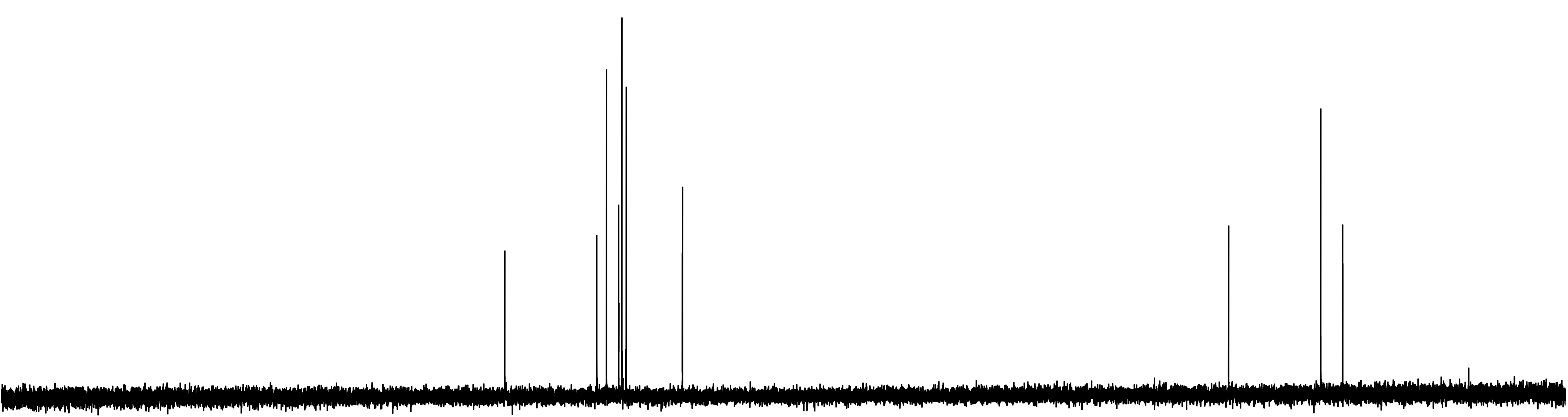

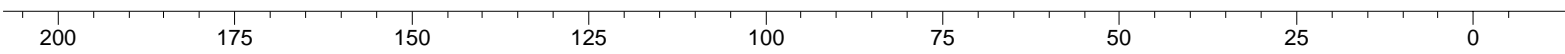




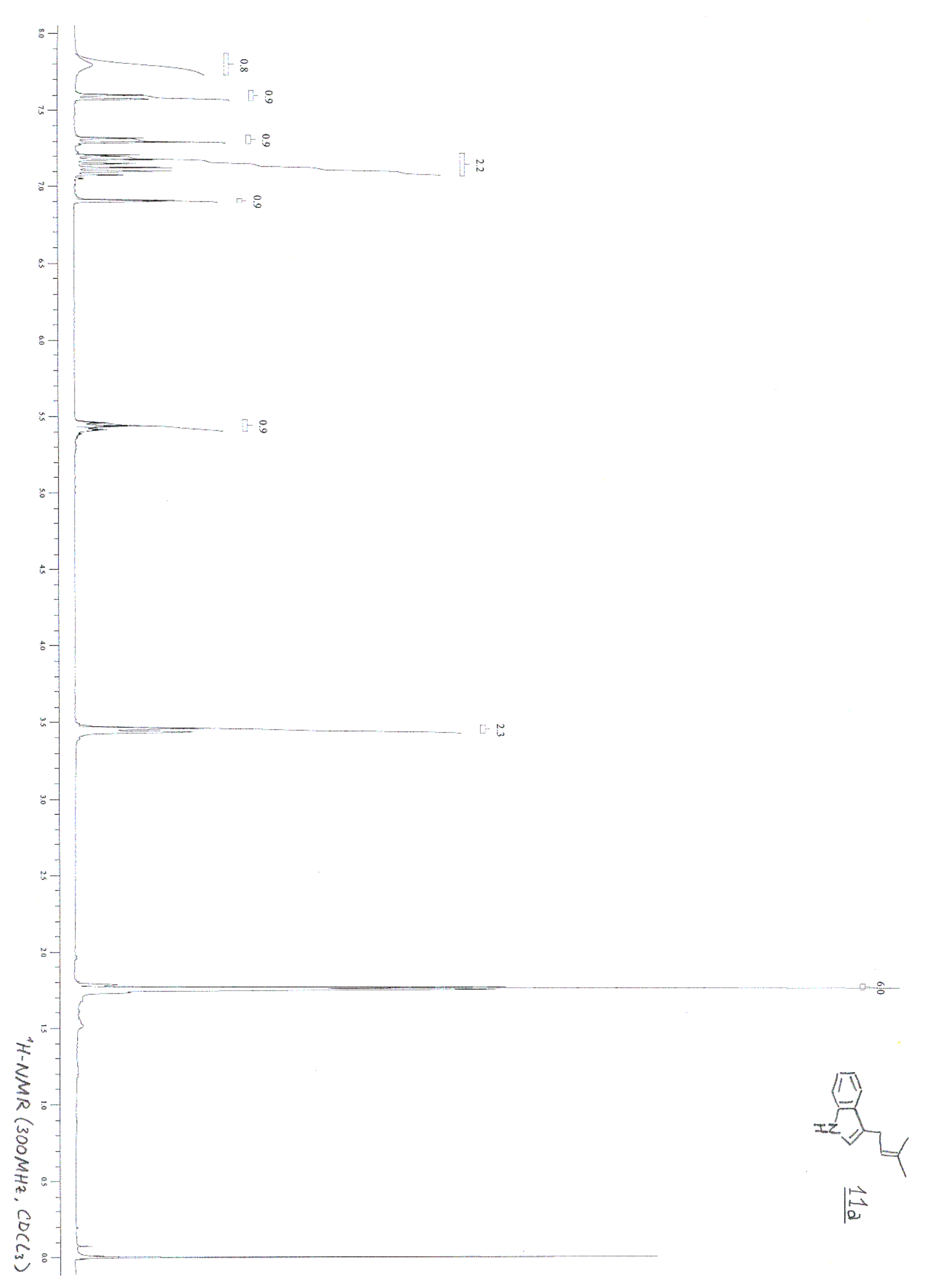




$$
\mid
$$



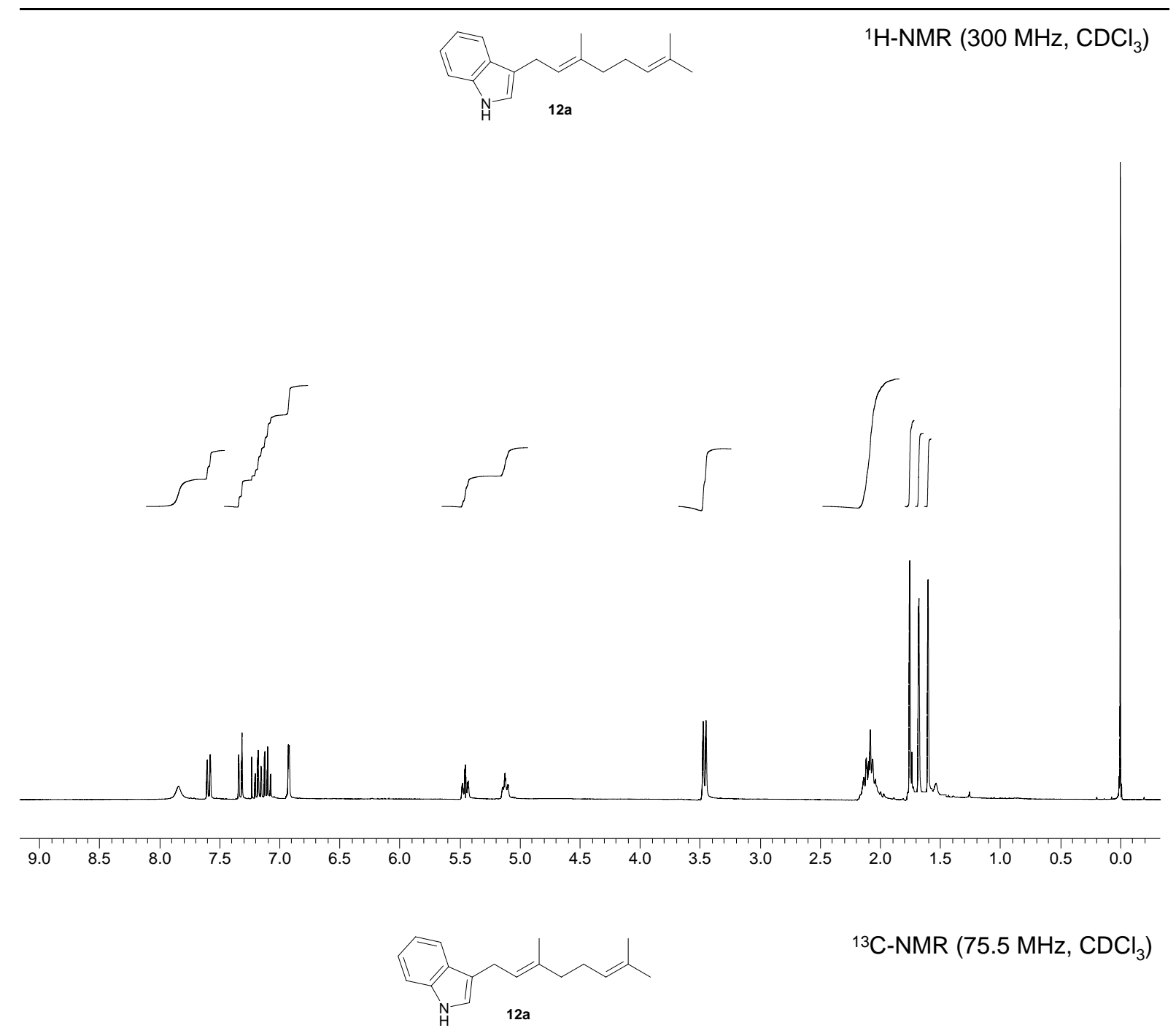

${ }^{13} \mathrm{C}-\mathrm{NMR}\left(75.5 \mathrm{MHz}, \mathrm{CDCl}_{3}\right)$

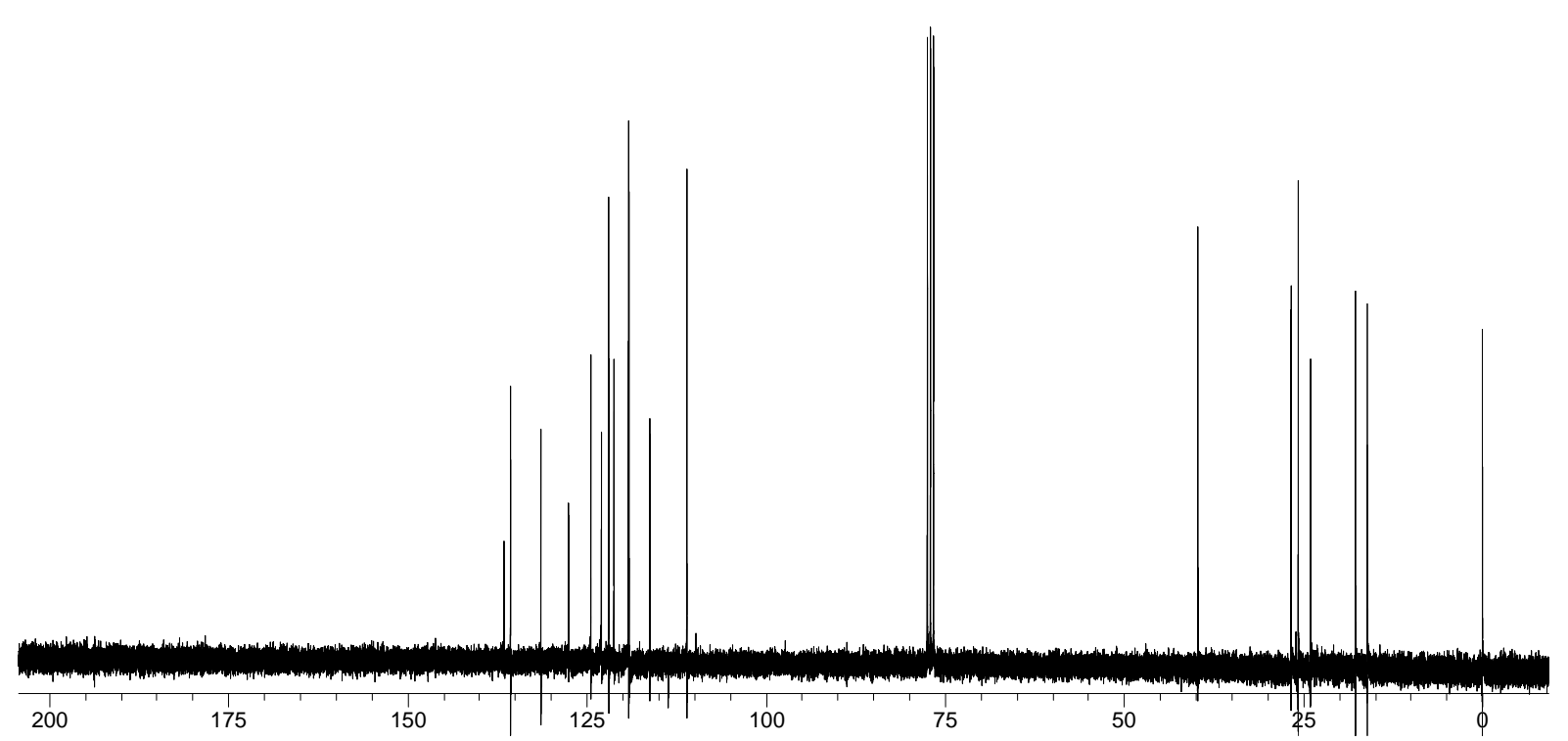



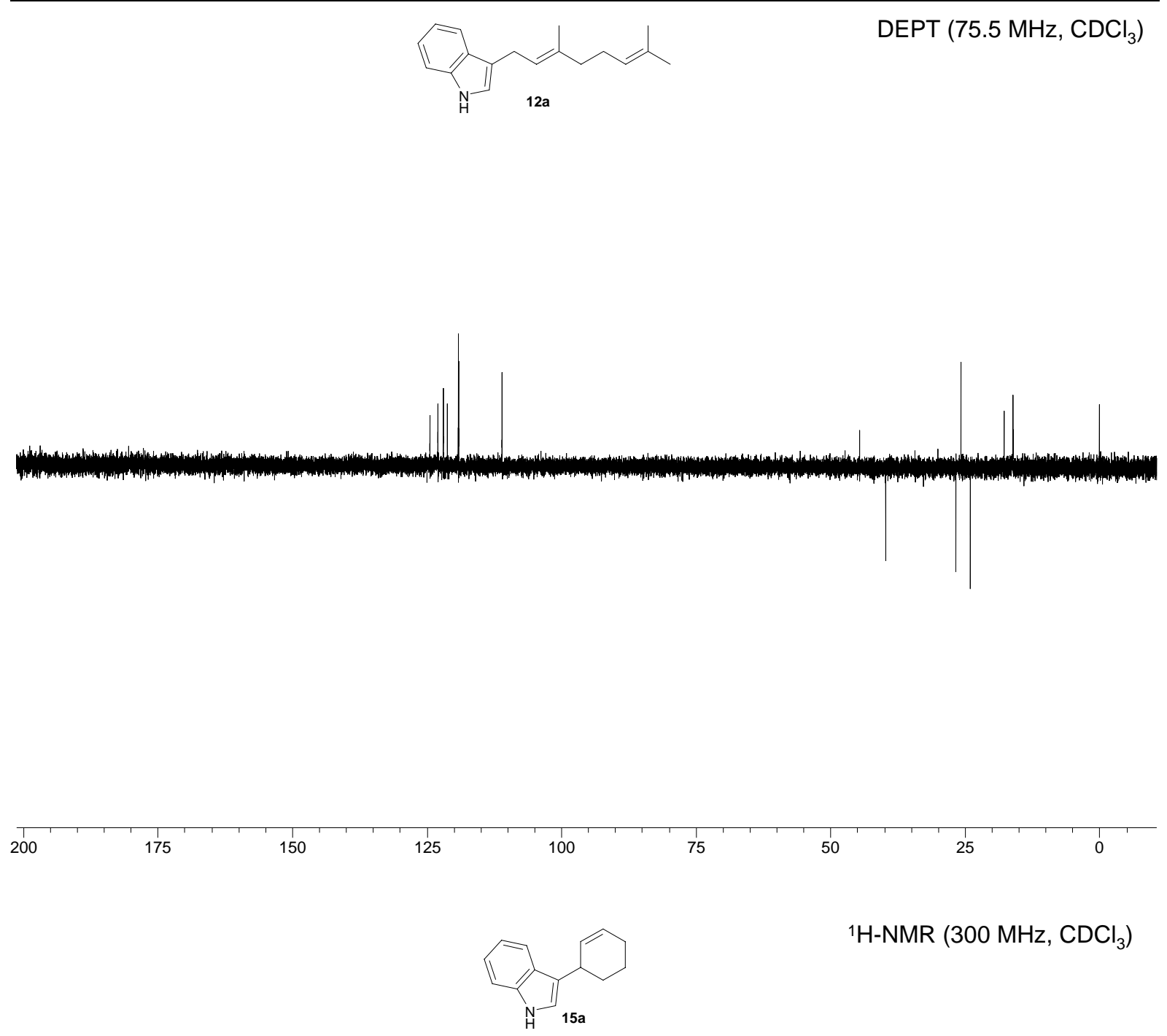

1H-NMR (300 MHz, $\mathrm{CDCl}_{3}$ )

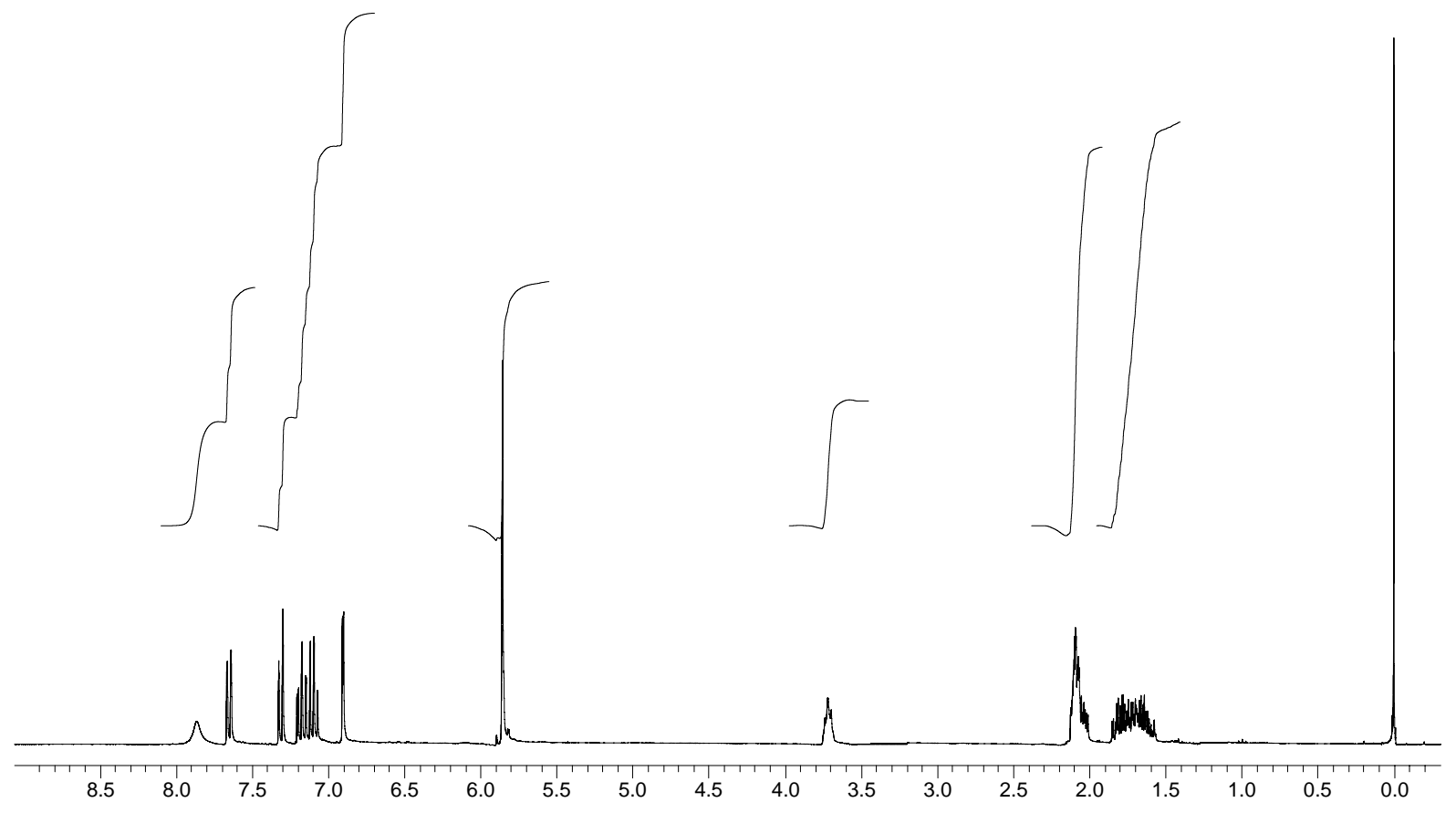

S15 

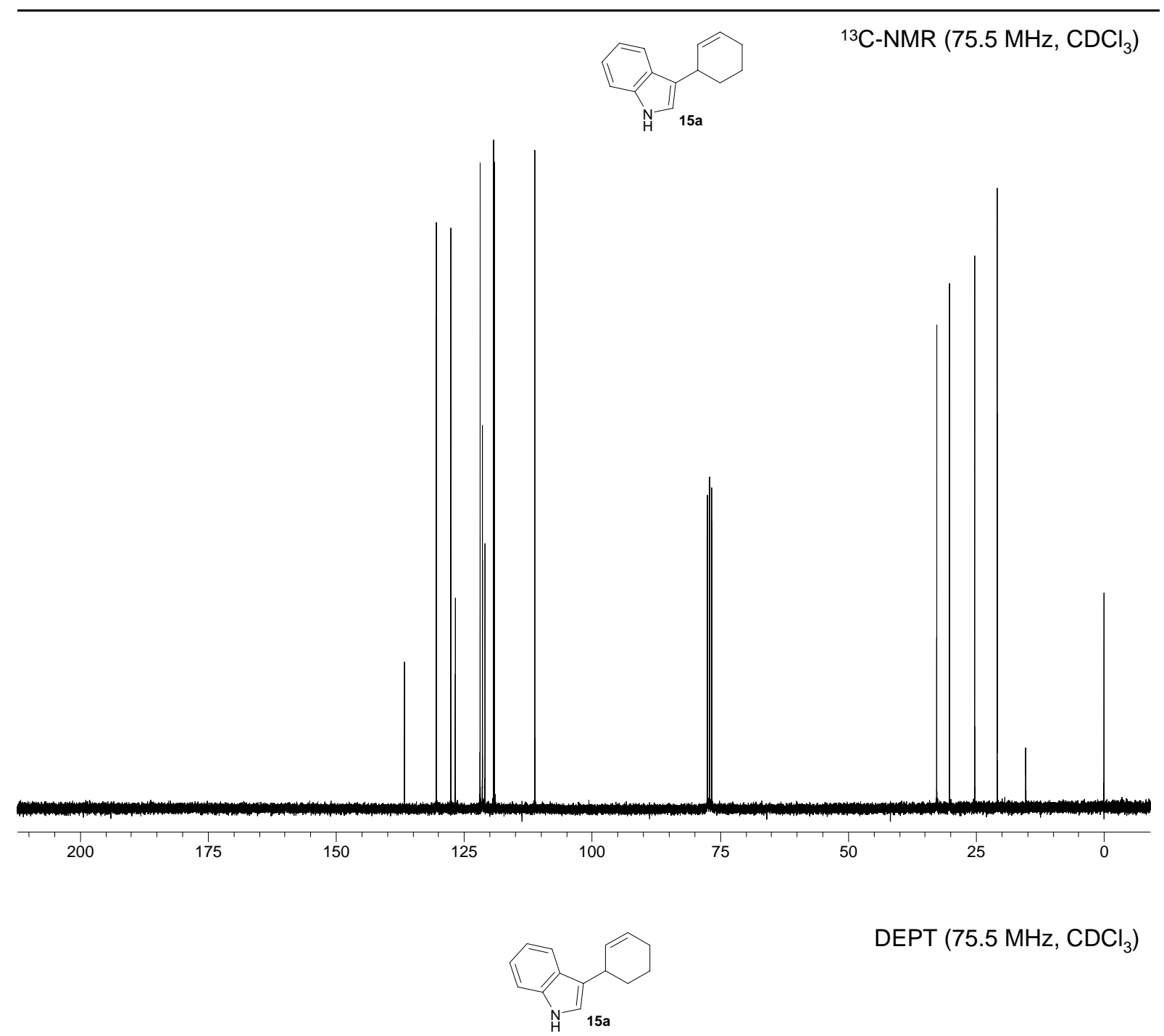

DEPT (75.5 MHz, $\left.\mathrm{CDCl}_{3}\right)$

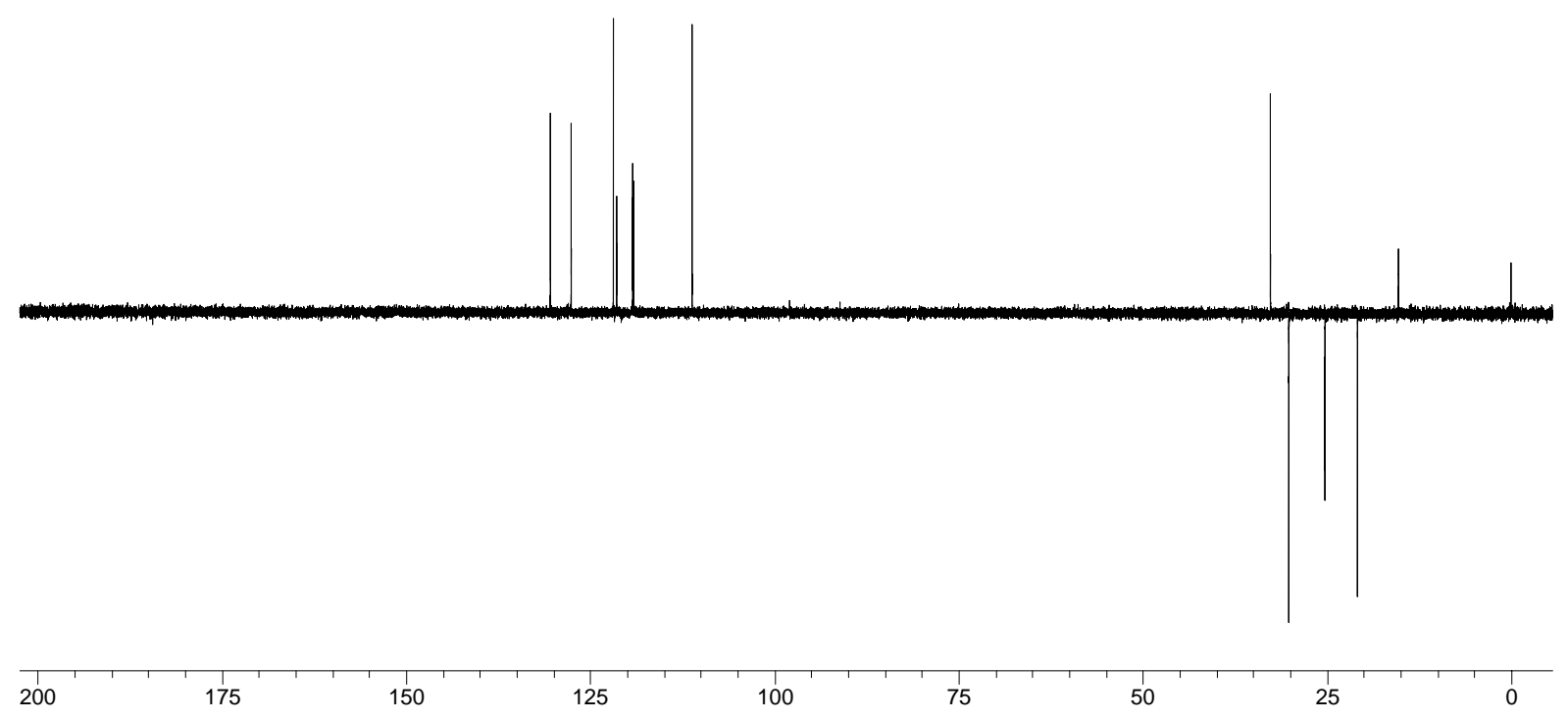



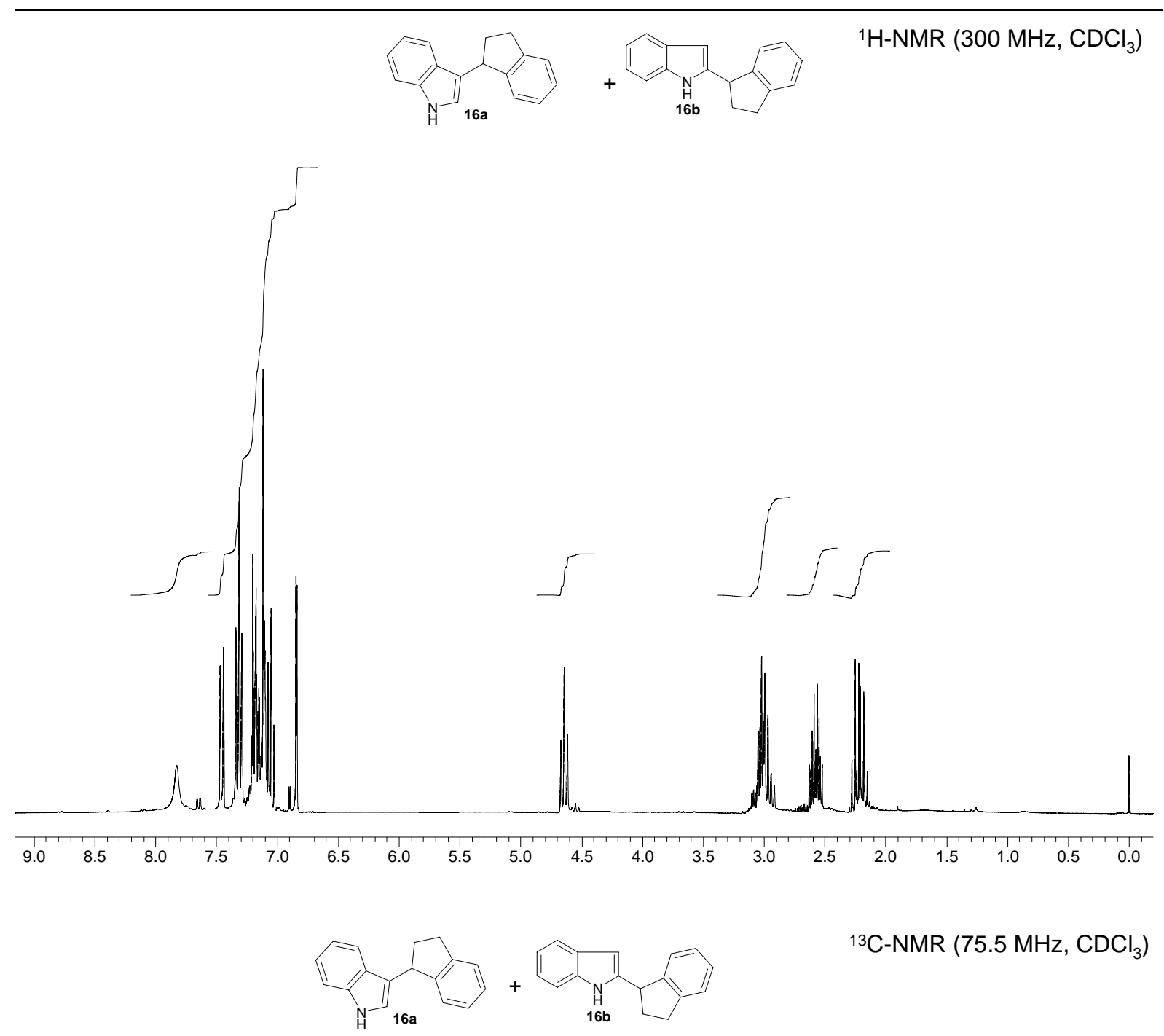

${ }^{13} \mathrm{C}-\mathrm{NMR}\left(75.5 \mathrm{MHz}, \mathrm{CDCl}_{3}\right.$ )

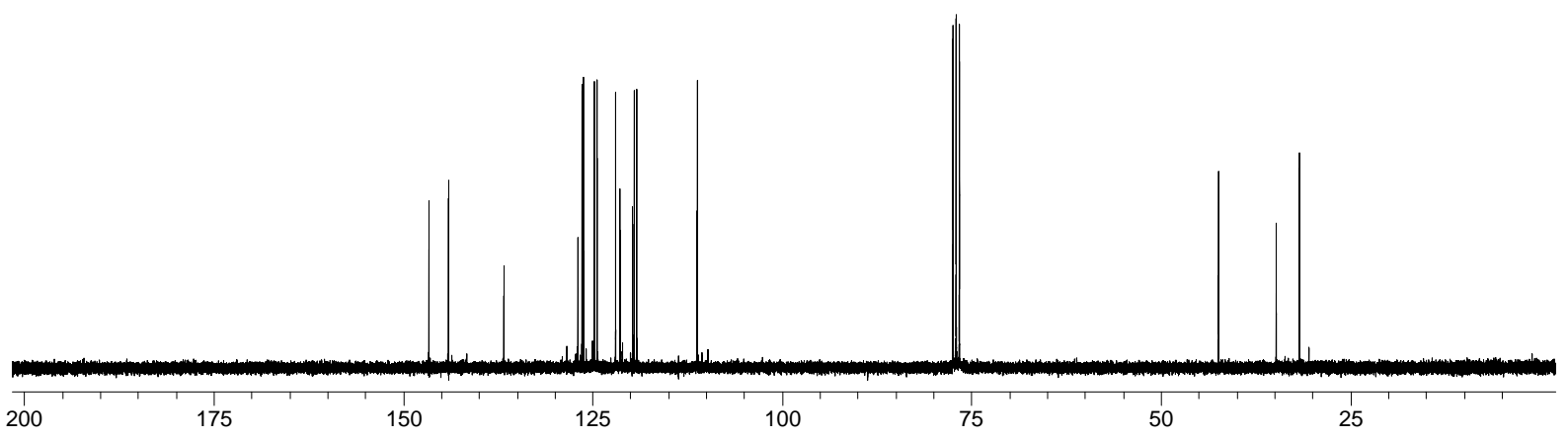




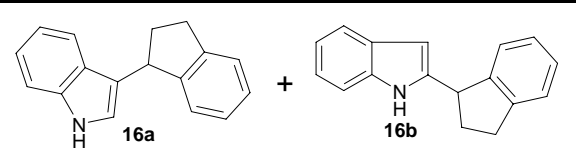

DEPT $\left(75.5 \mathrm{MHz}, \mathrm{CDCl}_{3}\right)$
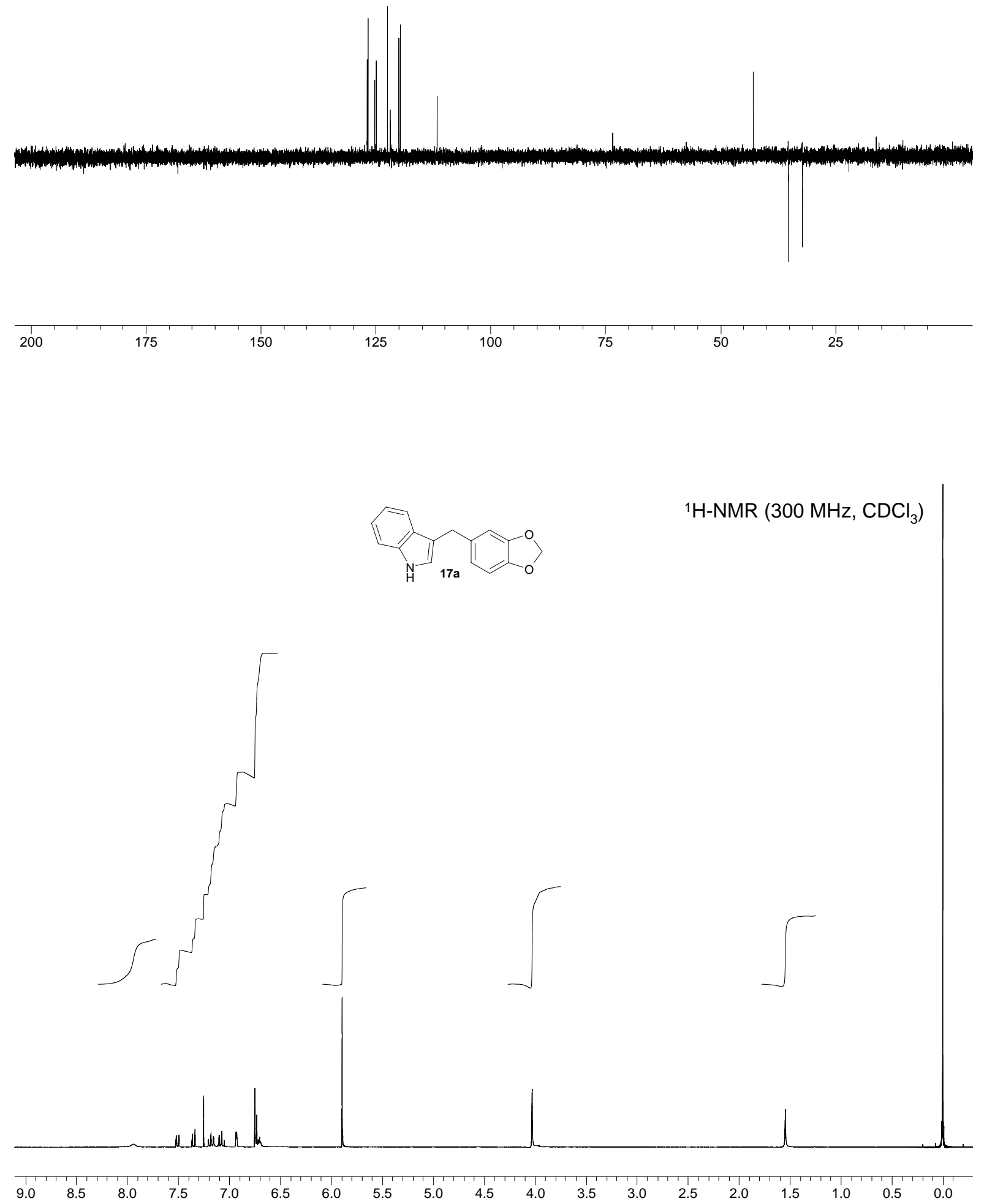

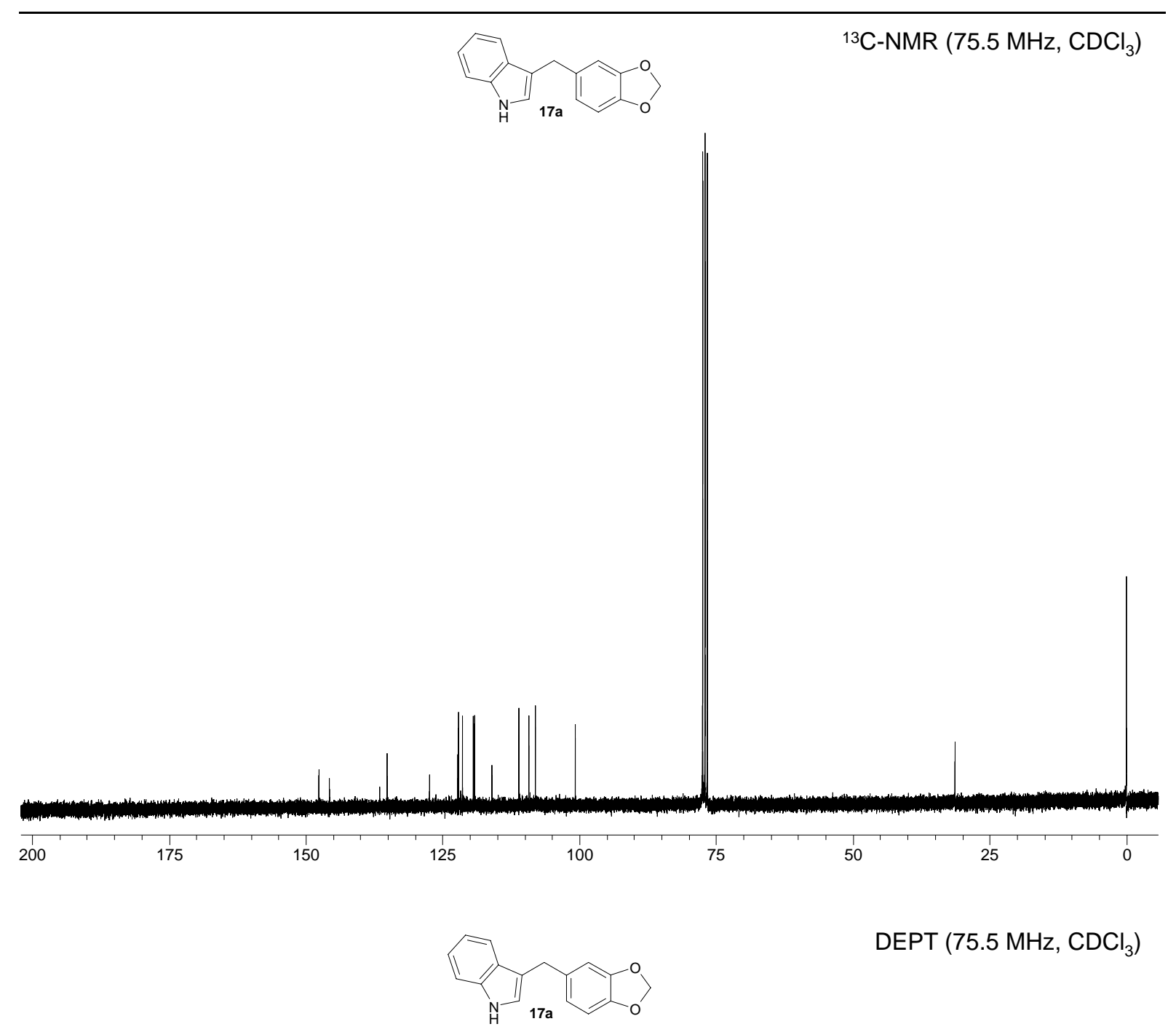

DEPT (75.5 MHz, $\mathrm{CDCl}_{3}$ )

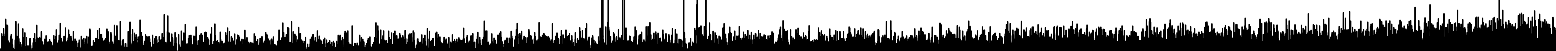

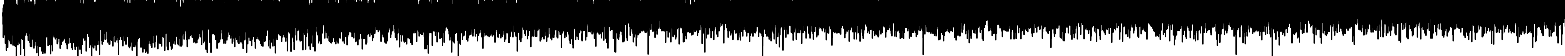

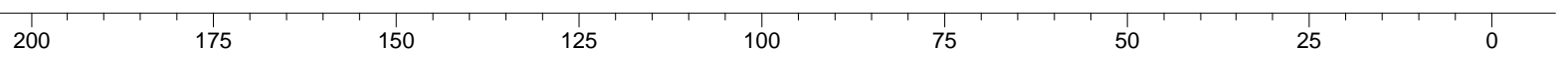



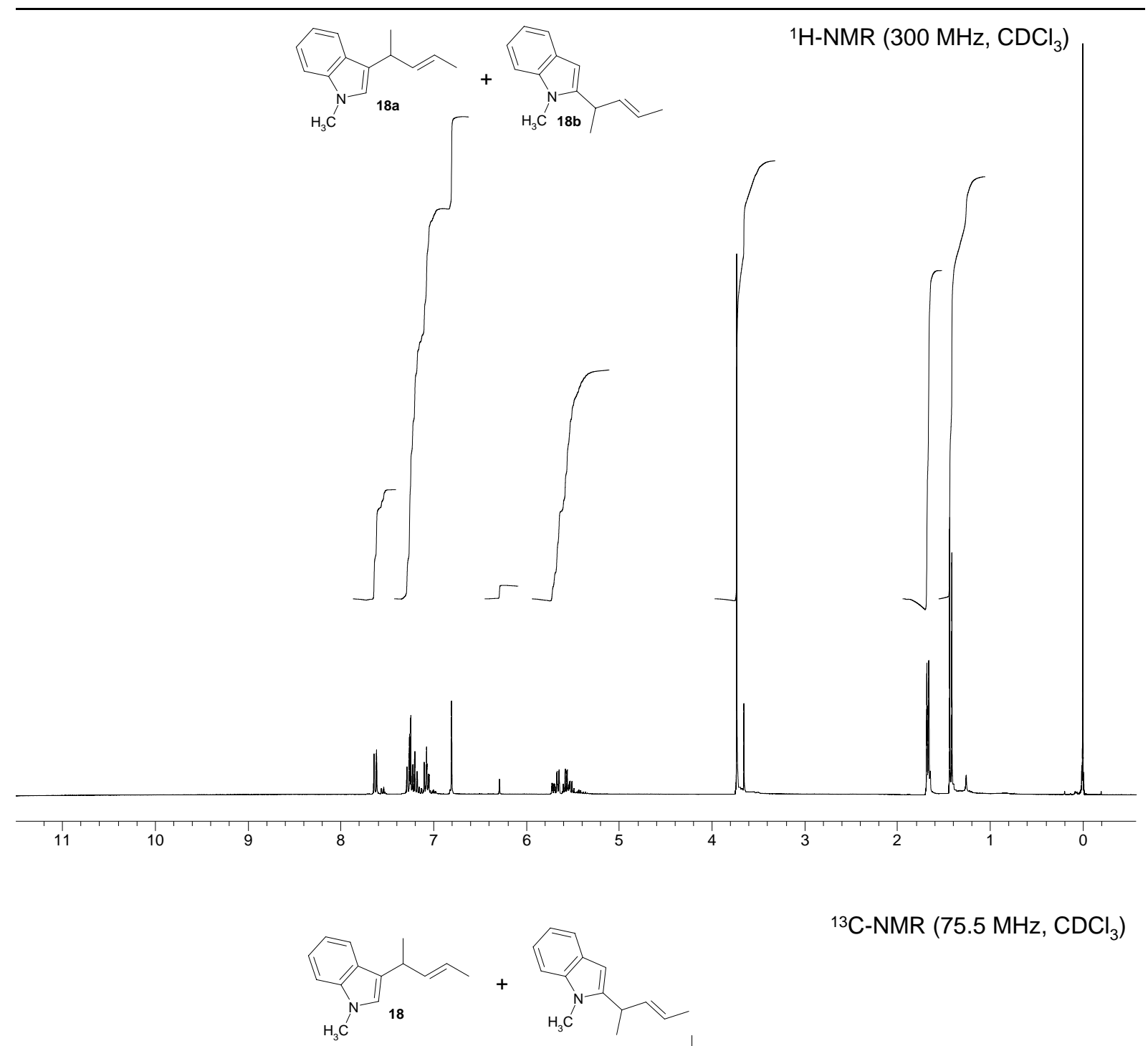

${ }^{13} \mathrm{C}-\mathrm{NMR}\left(75.5 \mathrm{MHz}, \mathrm{CDCl}_{3}\right.$ ) 


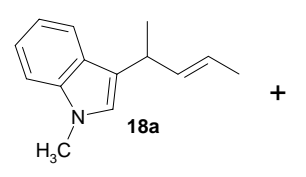

DEPT (75.5 MHz, $\mathrm{CDCl}_{3}$ )

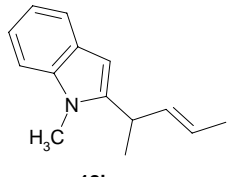

$18 b$

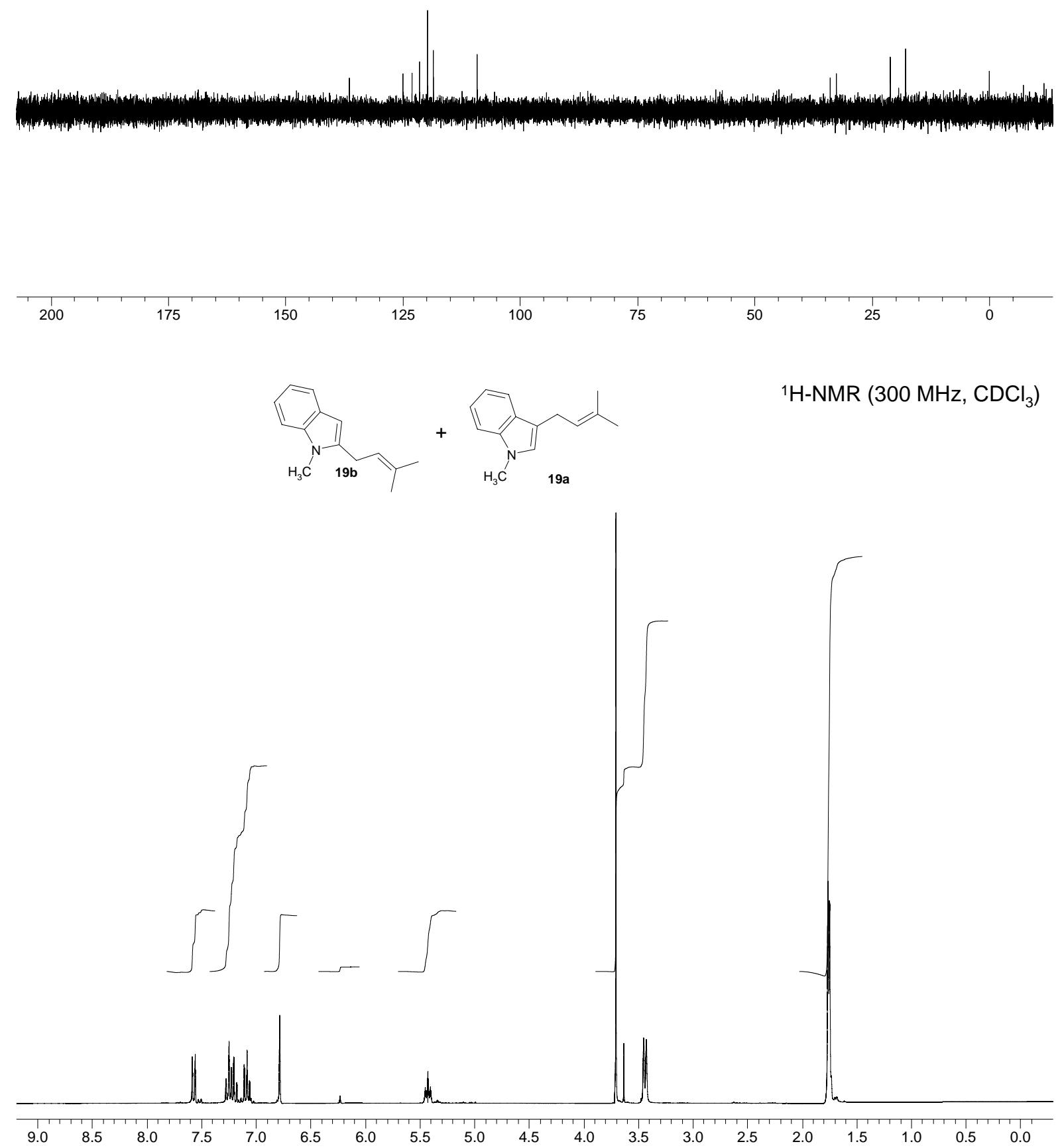



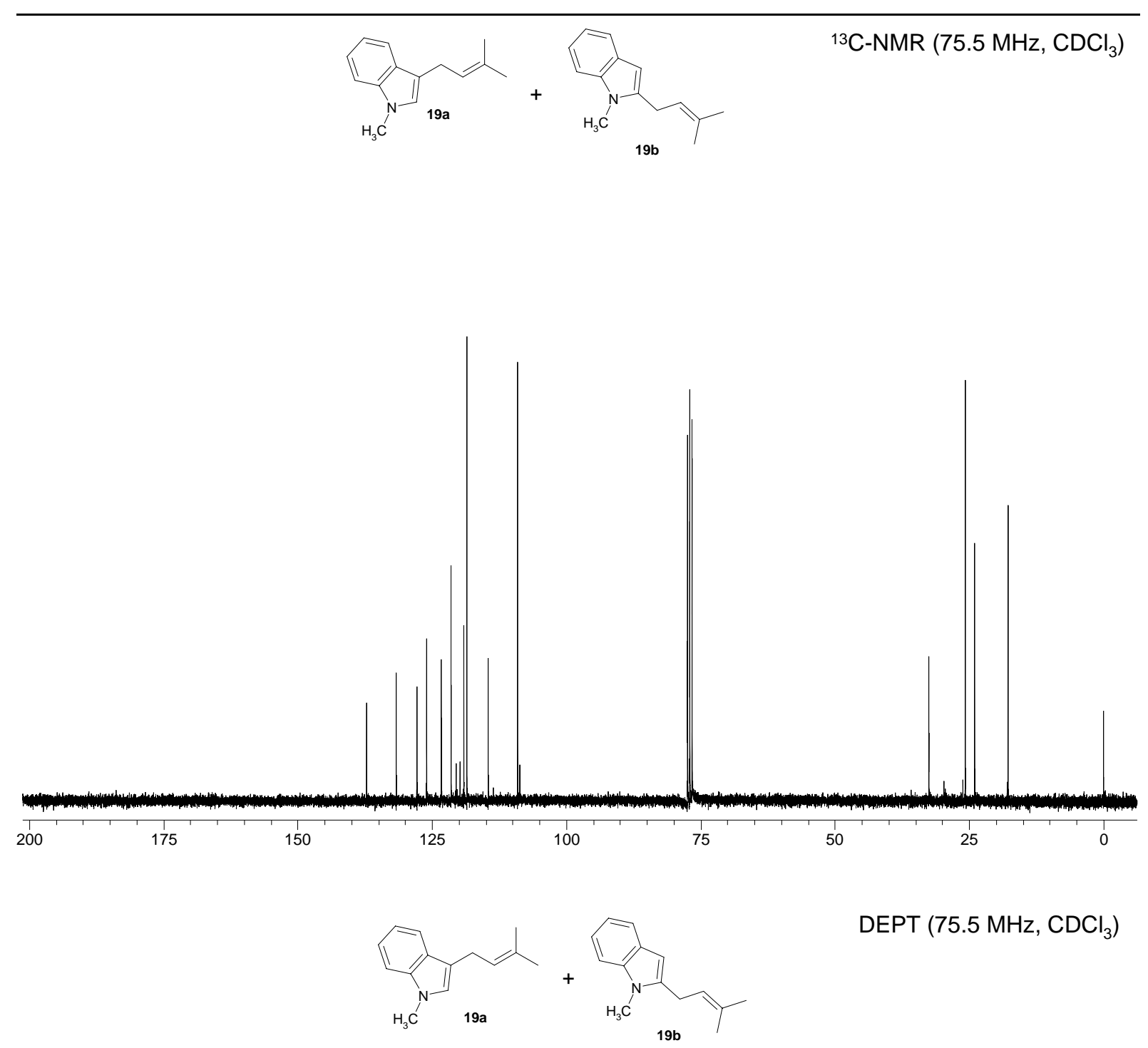

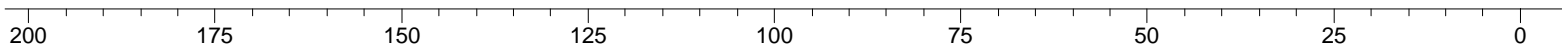



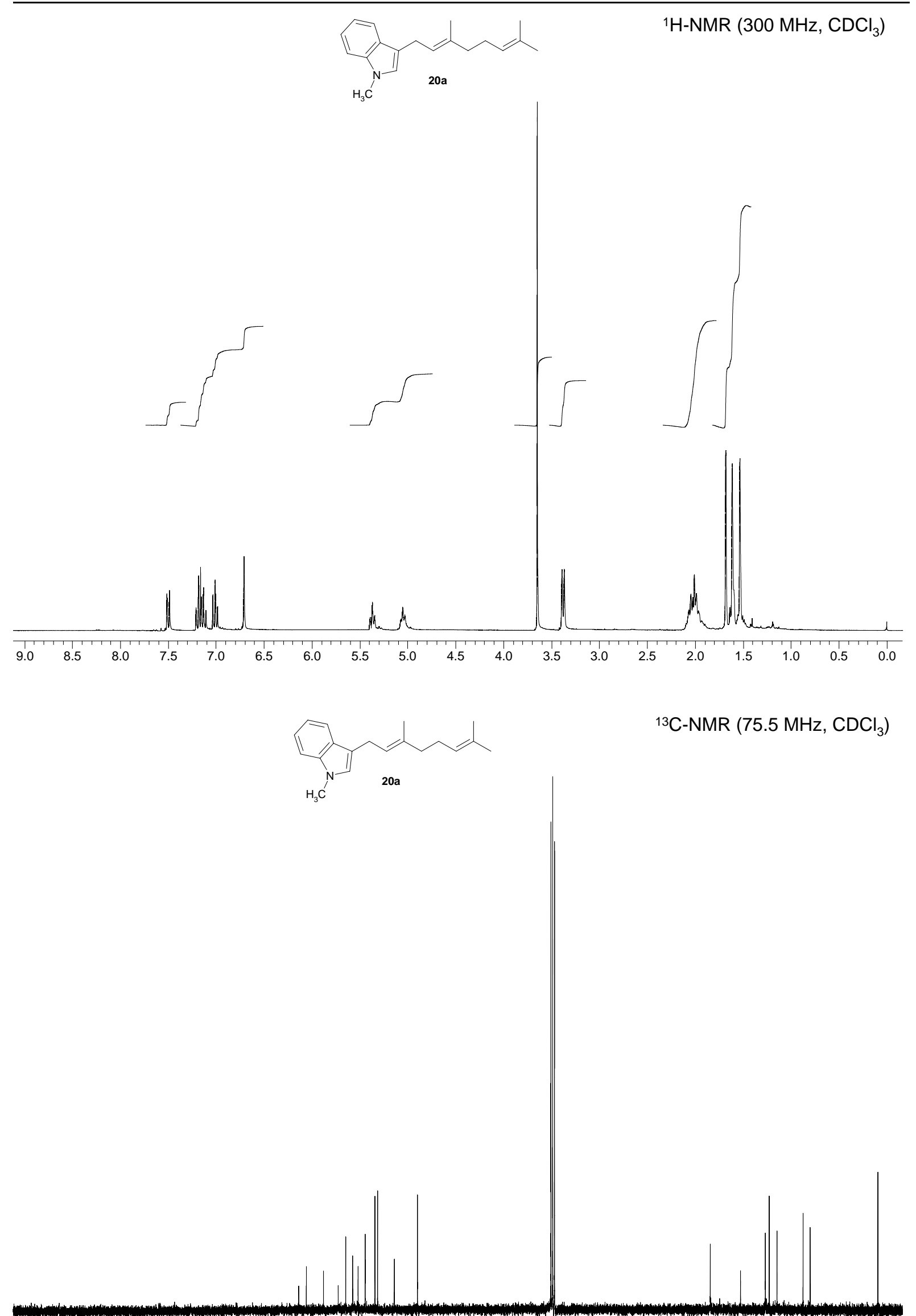

${ }^{13} \mathrm{C}-\mathrm{NMR}\left(75.5 \mathrm{MHz}, \mathrm{CDCl}_{3}\right)$

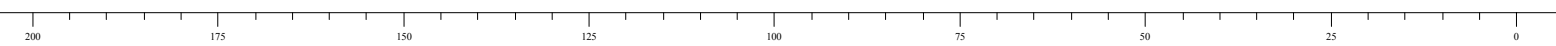




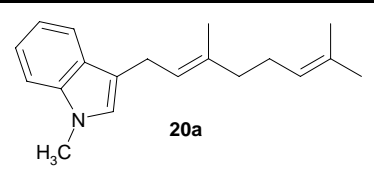

DEPT (75.5 MHz, $\mathrm{CDCl}_{3}$ )

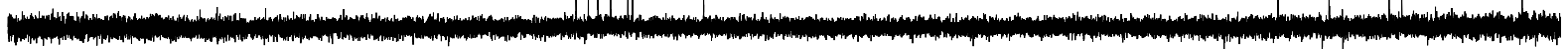

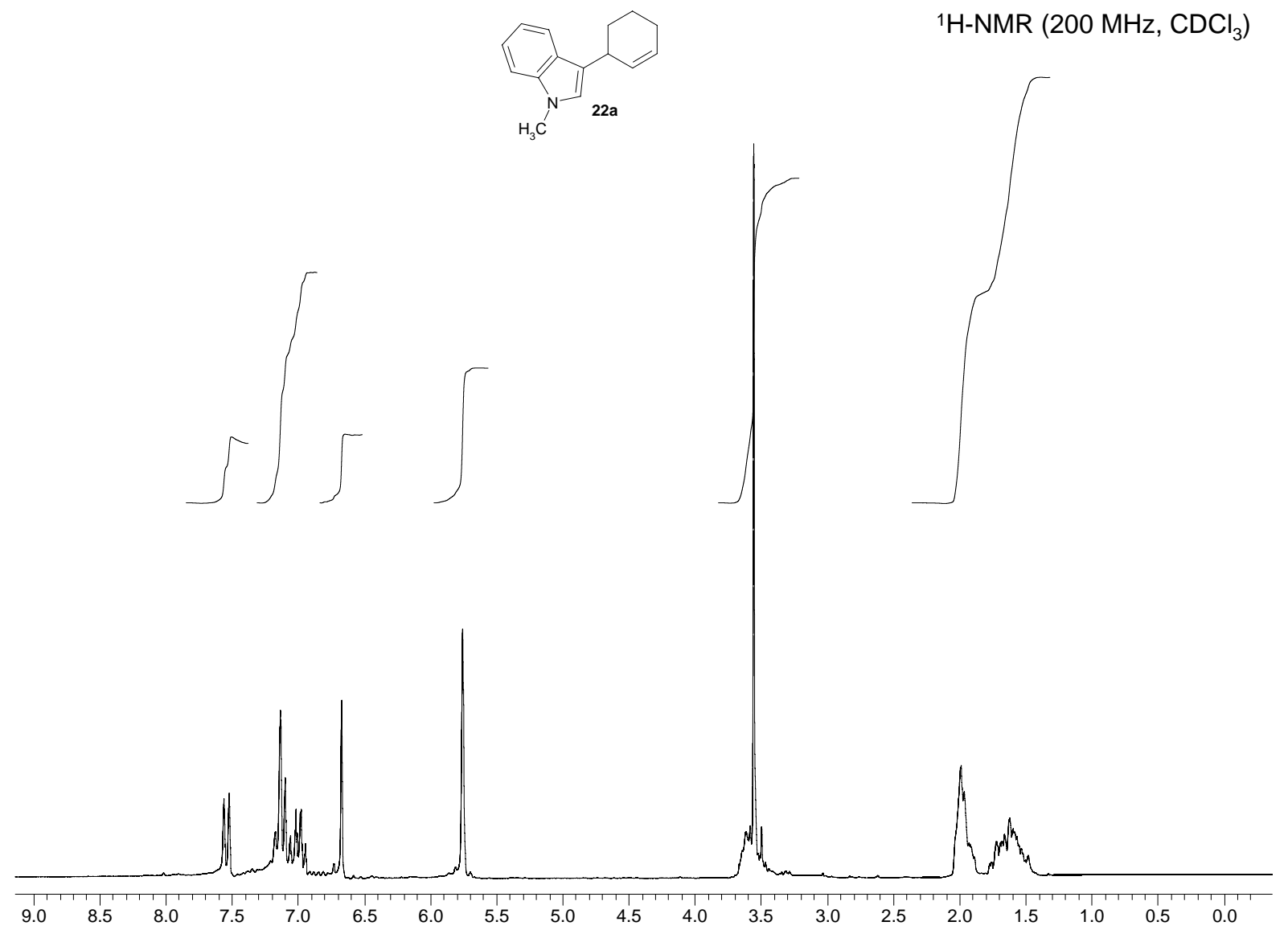



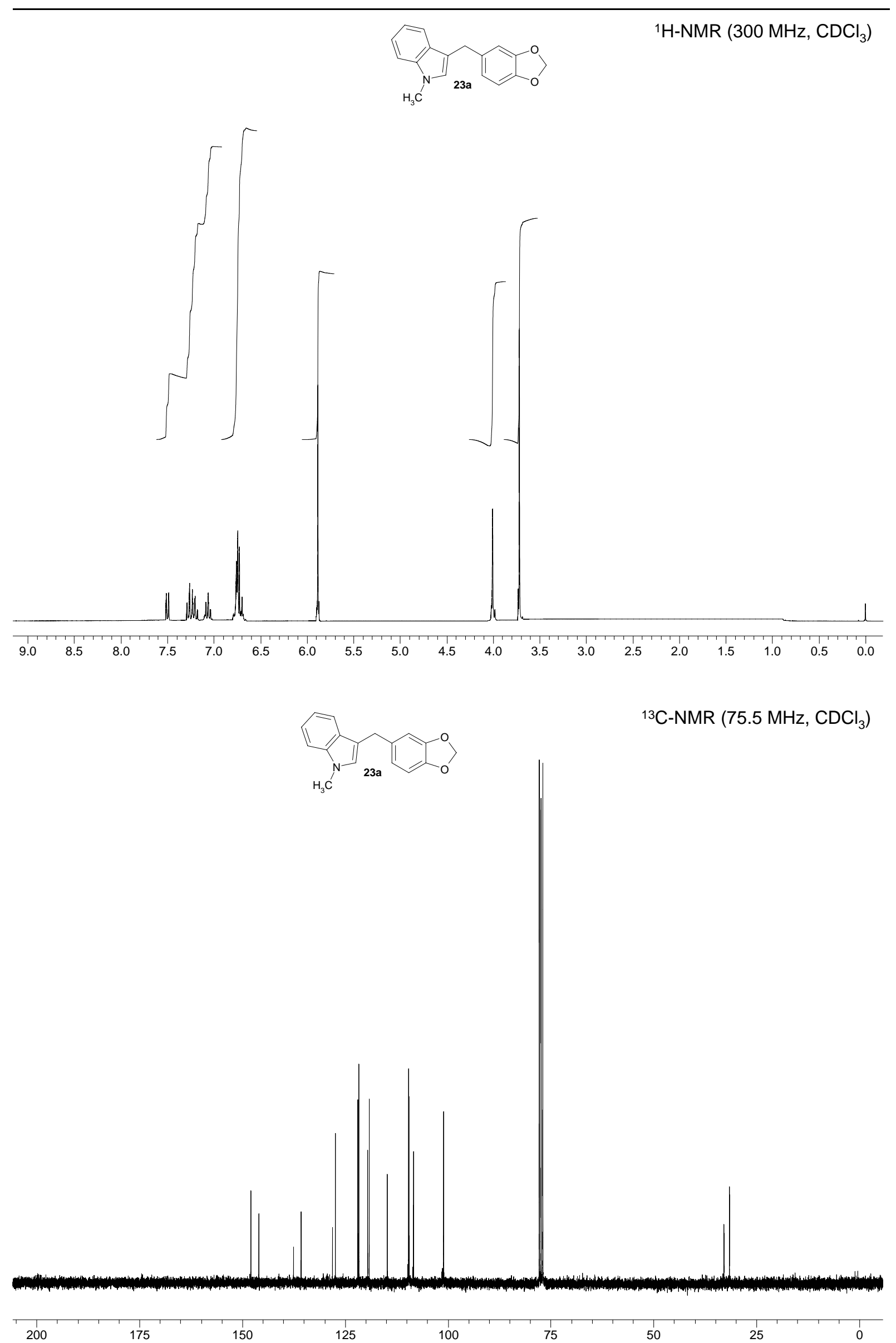


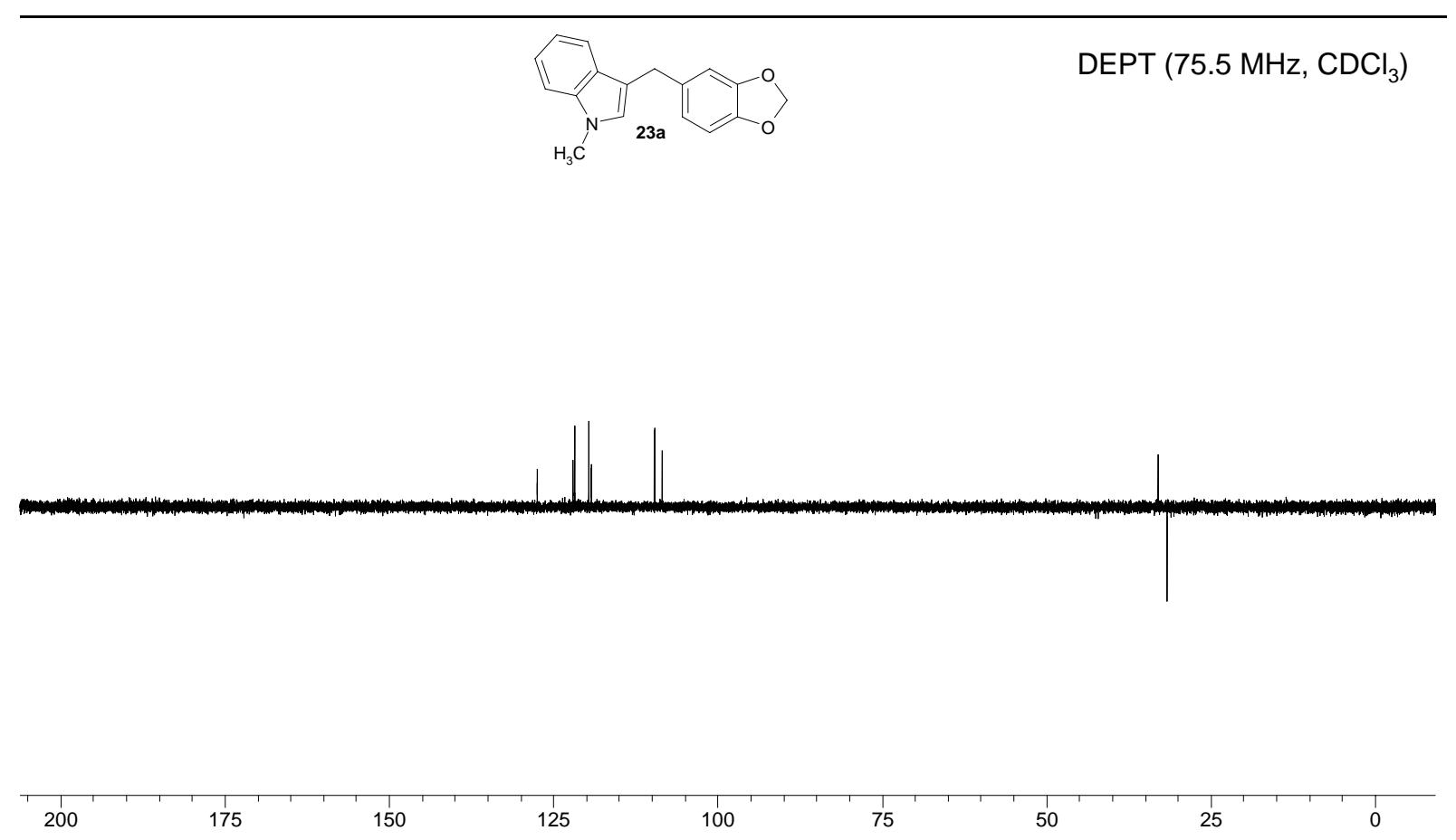

